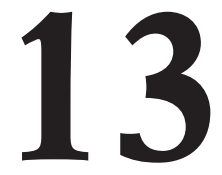

\title{
AULAS AFECTIVAS E INCLUSIVAS Y BIENESTAR ADOLESCENTE: UNA REVISIÓN SISTEMÁTICA
}

(EMOTIONAL AND INCLUSIVE CLASSROOMS AND ADOLESCENT WELL-BEING: A SYSTEMATIC REVIEW)

Patricia Iglesias-Díaz

Clara Romero-Pérez

Universidad de Sevilla

DOI: $10.5944 / e d u c X X 1.28705$

Cómo referenciar este artículo/How to reference this article:

Iglesias-Díaz, P., y Romero-Pérez, C., (2021). Aulas afectivas e inclusivas y bienestar adolescente: una revisión sistemática. Educación XX1, 24(2), 305-350. https://doi.org/10.5944/educXX1.28705

Iglesias-Díaz, P., \& Romero-Pérez, C., (2021). Emotional and inclusive classsrooms and adolescent well-being: a systematic review. Educación XX1, 24(2), 305-350. https://doi.org/10.5944/ educXX1.28705

\section{RESUMEN}

El ambiente de aula percibido por los estudiantes incide de forma significativa en su aprendizaje y desarrollo de la personalidad. El profesorado puede contribuir a promover el bienestar adolescente y la inclusión a través del clima emocional de aula. Los estudios sobre clima de aula, bienestar adolescente e inclusión en educación secundaria son minoritarios en comparación con los existentes en educación infantil y primaria. En este contexto, los objetivos de este trabajo son los siguientes: (a) analizar las relaciones entre un clima de aula afectivo e inclusivo con el bienestar adolescente; (b) sistematizar las competencias docentes más apropiadas para dinamizar la calidad afectiva e inclusiva del ambiente de aula en educación secundaria. 
Para responder a los objetivos del estudio, se ha llevado a cabo una revisión sistemática de la literatura y se han seguido las directrices marcadas en la declaración PRISMA para revisiones sistemáticas científicas. Para la revisión metodológica, se evaluaron los artículos con las herramientas JBI Critical Appraisal Tools. Se consultaron las bases de datos ERIC y Web of Science. El período de búsqueda comprendió desde 2016 a 2020.

La revisión se realizó sobre la base de 32 estudios seleccionados. Los resultados obtenidos ofrecen evidencias contrastadas del impacto de un ambiente afectivo e inclusivo de aula en el bienestar adolescente de educación secundaria. Asimismo, se demuestra el papel destacado del profesorado en la promoción de un ambiente amigable e inclusivo de aula y la necesidad de formación en competencias emocionales, interpersonales e interculturales. Se concluye que un clima de aula afectivo e inclusivo promueve no solo un desarrollo óptimo de la personalidad en la etapa de la adolescencia, sino que también impulsa la socialización en los valores de aprecio y respeto a la diversidad.

\section{PALABRAS CLAVE}

Ambiente de la clase, inclusión, bienestar del estudiante, enseñanza secundaria, competencias del docente

\section{ABSTRACT}

The classroom environment as perceived by students significantly impacts their learning and personality development. Teachers can help promote adolescent well-being and inclusion through the classroom environment. Studies on classroom climate, adolescent well-being and inclusion in secondary education are a minority compared to those in early childhood and primary education. In this context, the objectives of this work are the following: (a) to analyze the relationships between an emotional and inclusive classroom climate with the emotional well-being of adolescents; (b) to systematize the most appropriate teaching competencies to foster the affective and inclusive quality of the classroom environment in secondary education.

In order to respond to the study's objectives, a systematic review of the literature has been carried out and the guidelines marked in the PRISMA statement for systematic scientific reviews have been followed. For the methodological review, the articles were evaluated with the JBI Critical Appraisal Tools. The ERIC and Web of Science databases were consulted. The search period was from 2016 to 2020 .

The review was conducted on the basis of 32 selected studies. The results obtained offer contrasted evidence of the impact of an affective and inclusive 
classroom environment on adolescent well-being in secondary education. It also demonstrates the prominent role of teachers in promoting a friendly and inclusive classroom environment and the need for training in emotional, interpersonal and intercultural competences. It is concluded that an emotional and inclusive classroom climate promotes not only optimal personality development in the adolescent stage, but also encourages socialization in the values of appreciation and respect for diversity.

\section{KEY WORDS}

Classroom environment, inclusion, student welfare, secondary education, teacher competencies

\section{INTRODUCCIÓN}

Asegurar una enseñanza equitativa y de calidad en las etapas primaria y secundaria constituye una de las metas educativas incluidas en el nuevo contrato social global de la Agenda 2030. Desde la perspectiva de la profesión docente, esta enseñanza equitativa y de calidad se orienta al desarrollo de las competencias para el trabajo y para la vida de todo el alumnado sin excepción, para que puedan integrarse con éxito en un entorno cada vez más competitivo. Un entorno que genera oportunidades pero que también eleva barreras especialmente entre aquellos grupos de alumnos o colectivos más vulnerables, con una clara incidencia en su bienestar emocional. Los centros educativos pueden contribuir a desarrollar la resiliencia, el bienestar psíquico y la inclusión de los escolares más vulnerables a través del ambiente de aprendizaje (European Commission, 2019).

La construcción de estos ambientes educativos precisa de estrategias sistémicas a nivel macro, con incidencia en la dinámica de los propios centros educativos y a nivel micro, con repercusiones en la dinámica de aprendizaje que se propician en el interior de las aulas. Es este último nivel, el que se ha acotado como núcleo de interés en este artículo. No cabe duda que el aula es el escenario de acción del que, con mayores posibilidades de éxito, dispone el profesorado para transformar el ambiente de aprendizaje, hacerlo pivotar sobre el principio de inclusión y orientarlo hacia el bienestar psíquico de los escolares. Es por ello que entendemos que los vínculos entre la calidad de las experiencias emocionales vividas en las aulas por los escolares (aulas afectivas), la participación, apoyos y vinculación de todos los estudiantes (aulas inclusivas) y el bienestar psíquico de los escolares son muy estrechos, como demuestran los estudios recopilados en esta revisión sistemática. En este sentido, se sostiene en estas páginas que la calidad afectiva del ambiente de aprendizaje, constituye un prerrequisito básico para generar ambientes inclusivos de aprendizaje que redunda, en última instancia, en el bienestar psíquico de todos los estudiantes. 
Gestionar adecuadamente el ambiente de aprendizaje constituye una competencia profesional en la profesión docente. Aprender a generar las condiciones imprescindibles para el adecuado desarrollo de los procesos de enseñanza-aprendizaje, a la vez que se propician las competencias socioafectivas y personales del alumnado, de acuerdo con las necesidades y características individuales del mismo, es una competencia esencial que el profesorado ha de movilizar a diario en su contexto de práctica. La naturaleza interactiva e interpersonal del quehacer docente convierte al profesorado en un dinamizador de la vida colectiva en el aula y en el centro, construyendo a través de las dinámicas relacionales que se generan en el interior del aula, un clima de aprendizaje óptimo, estimulante y acogedor. En este artículo, y basándonos en la literatura científica, los términos ambiente o clima de aula se emplean como conceptos sinónimos. Ambos hacen referencia a las condiciones académicas y socio-emocionales del aula que influyen en las situaciones de aprendizaje. Se conceptualiza como un sistema dinámico que incluye tanto la conducta del docente como las interacciones profesorado/estudiante y las interacciones entre los propios alumnos. De acuerdo con los instrumentos de evaluación usualmente empleados para evaluar la calidad del clima de aula (Cimpian et al. 2021), y a pesar de la diversidad de aproximaciones teóricas al constructo, se puede afirmar que se compone de varias dimensiones (Moos y Trickett, 1974; Fraser, 1998) que incluye dos niveles: clima académico (orientación a la tarea, estilo de enseñanza investigador) y clima emocional (equidad, relaciones positivas y de apoyo, participación, cooperación).

El ambiente de aula percibido por los estudiantes influye de forma significativa en sus aprendizajes y en el desarrollo de su personalidad. El clima de aula está estrechamente relacionado con la dinámica motivacional de los estudiantes (Viau, 2009). De acuerdo con Doyle (1986) y Archambault et Chouinard (2016) el indicador de un ambiente óptimo de aprendizaje se observa a partir del grado de cooperación y sentimientos de pertenencia que presiden las relaciones entre los propios estudiantes y entre estos y el profesorado. Cuando los estudiantes perciben sentirse como miembros de la comunidad educativa y del grupo-clase de referencia, las probabilidades para "dejarse educar", aprender de modo autónomo y experimentar emociones placenteras aumentan. No en vano, una de las razones por las que a los estudiantes les gusta asistir a sus clases y aprender en ellas es por el clima positivo que viven en las aulas (Sieberer-Nagler, 2015). Además, en la medida en que los aprendices se sienten seguros, integrados, apoyados y conectados afectivamente con sus iguales y sus profesores, el rendimiento académico aumenta. De hecho, estos hallazgos han sido clarificados por multitud de estudios a nivel internacional (Lee y Smith, 1999; Sherblom et al., Marshall y Sherblom, 2006; Whitlock, 2006; Shindler et al., 2016; Nisar et al. 2017; Voight y Hanson, 2017; Fakunle y Ale, 2018).

De acuerdo con recientes estudios, la calidad del ambiente de aula decrece a medida que los estudiantes promocionan de la etapa primaria a la secundaria (Shindler et al., 2016). También decrece entre el profesorado de secundaria el 
foco de interés relacional. Si en la educación primaria el profesorado privilegia más al alumno, singularmente considerado, y se preocupa por satisfacer las necesidades psicológicas de este, en la educación secundaria el profesorado suele exhibir un estilo relacional centrado en el grupo y se centra más en satisfacer las necesidades académicas, que en atender a las necesidades psicoafectivas de estos (Kalin et al., 2017).

Los beneficios generados por un ambiente de aula positivo van más allá de sus efectos en el desarrollo cognitivo y emocional o académico de los escolares. Un clima afectivo en el aula cumple un rol compensador, en muchos casos, que contrarresta las posibles influencias negativas del entorno vital y sociocultural de los estudiantes. En un ambiente de aula cálido, autorizativo e inclusivo, los escolares perciben seguridad y sentimiento de pertenencia. La población escolar más vulnerable, expuesta a factores de riesgo por razones socioeconómicas, culturales o familiares son más susceptibles que los restantes estudiantes de sufrir procesos de exclusión en el entorno escolar (Romero-Pérez et al., 2019). El profesorado que proporciona un ambiente de tolerancia, comprensión y aceptación, suscita sentimientos de seguridad, apoyo y reconocimiento entre sus estudiantes (Bulut e Iflazoglu, 2016; Gizir, 2019).

Cuanto más alta es la seguridad psicológica del ambiente escolar, más bajos son los niveles de ansiedad estudiantil (Kulikova y Maliy, 2017). Asimismo, cuando los escolares perciben un ambiente de reconocimiento, apoyo y respeto, existe una mayor probabilidad de que estos exhiban estrategias adaptativas en el manejo del estrés y empleen una amplia gama de recursos personales con los que afrontar los retos que se presentan en sus contextos vitales (Newland et al., 2019). Es por ello que, en un estudio centrado en determinar los beneficios de un ambiente de aula respetuoso e inclusivo, se concluyó que los estudiantes que perciban tales cualidades, sus comportamientos denotaron mayor predilección por el manejo de las emociones y frustraciones de carácter interno (Yeager, 2017). Por último, los entornos positivos de aula promueven la satisfacción vital, alientan la autoeficacia e impulsan cotas mayores de cohesión grupal en los escolares (Ghaith, 2003; Naghsh et al., 2017).

En los últimos años, se ha revitalizado el estudio del clima de aula, muy especialmente, en educación secundaria. Son varias las razones que explican este interés. En primer lugar, por las transformaciones en la profesión educativa (Vera Vila, 2019). El paradigma educativo centrado en el aprendizaje exige transformaciones tanto de mirada como en la práctica docente. En segundo lugar, por los propios cambios generacionales que se observa en el alumnado. Los estudiantes adolescentes de hoy aprecian que sus profesores los valoren por su sensibilidad y simpatía, sus habilidades sociales, responsabilidad y solidaridad (Berríos-Valenzuela y Buxarrais-Estrada, 2013). En tercer lugar, por el interés que concitan la salud mental infantil y adolescente y la calidad de los entornos educativos en los que se socializan (Thapa, 2013). De ahí que aprender a dinamizar ambientes escolares afectivos e inclusivos constituya uno de los 
desafíos actuales en la profesión educativa, muy especialmente en la educación secundaria por la complejidad psicopedagógica que conlleva esta etapa y por la importancia que cobra en la adolescencia el andamiaje emocional que brinde el profesorado (Yeager, 2017).

\section{El presente estudio, que aplica la metodología de revisión sistemática de la literatura, plantea dos objetivos:}

a) Analizar las relaciones entre un clima de aula afectivo con ambientes de aprendizaje inclusivos y el bienestar emocional de los adolescentes.

b) Sistematizar las competencias docentes más apropiadas para dinamizar la calidad afectiva del ambiente de aula en pos de la inclusión educativa y el bienestar emocional de los estudiantes de educación secundaria.

\section{Vinculados a estos objetivos, esta revisión se ha propuesto abordar las siguientes cuestiones de investigación:}

a) ¿Qué relaciones se dan entre un clima afectivo de aula y el bienestar adolescente de educación secundaria?

b) ¿Qué relaciones se dan entre un clima inclusivo de aula y el bienestar adolescente de educación secundaria?

c) ¿Qué competencias docentes han demostrado ser más apropiadas para promover la calidad afectiva e inclusiva del ambiente de aula en educación secundaria?

En este estudio se parte de la única revisión sistemática realizada hasta el momento, liderada por Kutsyuruba et al. (2015) en las que se exploran las relaciones entre clima escolar, seguridad, rendimiento académico y bienestar emocional de los estudiantes. El estudio consideró como muestra, investigaciones publicadas de 1963 a 2013. En dicho estudio, de carácter más comprensivo, se optó por no diferenciar las etapas de educación infantil, primaria y secundaria. Si se atiende a las demandas formativas del profesorado de la etapa secundaria, de acuerdo con la European Commission, the Education, Audiovisual and Culture Executive Agency (EACEA) y Eurydice (2019) estas suelen recaer en tres: la necesidad de movilizar las competencias interculturales, emocionales e interpersonales. No es de extrañar, dado que dos de los principales retos a los que han de responder los profesionales docentes son precisamente los desafíos de la atención a la diversidad y de la 
personalización de los ambientes de aprendizaje. Por tanto, entendemos que se hace necesaria una revisión sistemática de la literatura sobre el ambiente afectivo de aula y sus repercusiones en la inclusión educativa y el bienestar emocional de los estudiantes en esta etapa.

\section{METODOLOGÍA}

Para dar respuesta a las cuestiones formuladas y lograr los objetivos de investigación perseguidos se ha llevado a cabo una revisión sistemática de la literatura (Sánchez-Meca, 2010; Gouch et al., 2017; Newman y Gough, 2020; Zawacki-Ritcher et al., 2020).

Esta revisión sistemática se efectúa con el objeto de localizar, analizar y valorar aquellos estudios que analiza particularmente la dimensión socioafectiva del clima de aula, su impacto en la inclusión y sentimientos de pertenencia de los escolares y sus efectos en el bienestar emocional de los estudiantes de Educación Secundaria.

\section{Protocolo y registros}

El presente estudio ha seguido las directrices marcadas en la declaración PRISMA (Preferred Reporting Items for Systematic Reviews and Meta-Analyses) para revisiones sistemáticas científicas, con el propósito de preservar un desarrollo y una planificación metódica, a través de consideraciones metodológicas y ejemplificación de elaboraciones, en la presentación del informe (Hutton et al., 2015).

\section{Procedimiento}

Atendiendo al procedimiento y a las estrategias de búsqueda, se han consultado las bases de datos Education Resources Information Center (ERIC) y Web of Science (WOS). Se realizaron búsquedas de artículos científicos publicados desde 2016 a 2020, ambos inclusive. La búsqueda se realizó durante los meses de febrero a mayo de 2020.

\section{Estrategias de búsqueda}

En el proceso de búsqueda y selección se tuvieron en consideración las siguientes palabras clave: Educational Environment, Inclusion, School Climate, Well Being, Student Satisfaction, Student Welfare, Organizational Climate, Class Organization, Teacher Competencies, Mental Health, Teacher Student Relationship, Teaching Conditions, Teacher Influence y Psychosocial 
Development. Con el objeto de confinar a la búsqueda orden y estructuración, se utilizó como operadores lógicos las palabras "AND", "NOT" y "OR".

En el caso específico del portal Web of Science, se empleó una estrategia de rastreo diferente debido a la existencia de un sistema de búsqueda avanzada por pestañas desplegables. De este modo, primero se seleccionaron únicamente los artículos provenientes de la colección principal; una vez marcada la casilla relativa a documentos publicados en los últimos 5 años, se marcaron como índice de citas, las relacionadas con el Social Science Citation Index (SSCI) y Emerging Sources Citation Index (ESCI).

Con el fin de facilitar una mayor comprensión del contenido, el texto completo de los estudios seleccionados debía estar escrito en inglés o español, excluyendo aquellas publicaciones en idiomas diferentes a los mencionados. A su vez, en ambas bases de datos se utiliza como criterio de inclusión el nivel educativo referente a Educación Secundaria, quedando de esta forma excluidas las restantes etapas.

Por otro lado, y siguiendo los criterios de legibilidad empleados para la sistematización, se contemplaron estudios empíricos tanto de corte cualitativo como cuantitativo, incluyendo revisiones sistemáticas y descartando revisiones bibliográficas y narrativas.

Una vez introducidas las palabras clave y operadores boleanos, se establece otra casilla para la inclusión de los términos Secondary School, Lower Secondary General Education y Upper Secondary Education, cuyas búsquedas fueron independientes para evitar una reducción del área a investigar (Anexo: Tablas 1 y 2).

Para lograr el acceso completo a los estudios seleccionados, se empleó el catálogo Fama, perteneciente a la biblioteca de la Universidad de Sevilla. En aquellos casos en los que no existía disponibilidad en línea del documento, se hizo uso de los servicios telemáticos ofrecidos por la biblioteca, concretamente a la opción de préstamo interbibliotecario. Se solicitaron un total de 15 documentos.

\section{Selección de los estudios}

Una vez revisados el título y resumen de los artículos resultantes, así como eliminar las búsquedas duplicadas, quedaron un total de 204 artículos (143 de ERIC y 61 de WOS), los cuales fueron revisados para determinar el cumplimiento de los criterios de inclusión ya citados. Otro motor de búsqueda complementario ha sido The Global Science Gateway, portal con impronta internacional y pública de documentación científica, del cual se extrajeron artículos de revistas en inglés, por su difusión y validez a escala global. 


\section{Figura 1}

Diagrama de flujo PRISMA de la revisión sistemática. Adaptado de Moher et al., (2009)

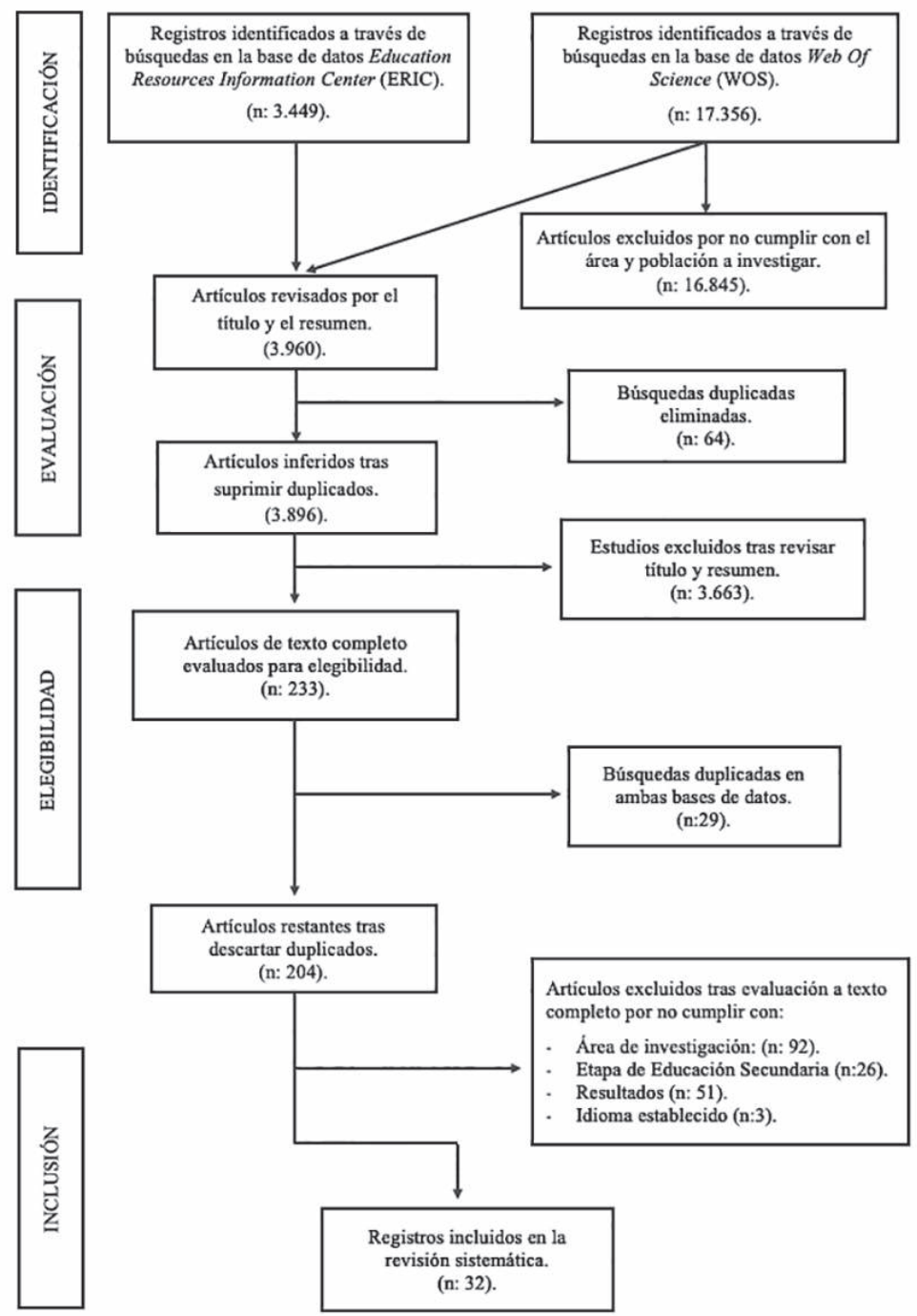

De esta forma, tras la fase de identificación y cribado, se realizó una lectura más detallada de todos los apartados que constituyen los artículos, aplicando nuevamente los criterios de inclusión y exclusión para la obtención de estudios en íntegra relación con nuestros objetivos. Como se puede ver en la Figura 1, el resultado final tras el proceso genera un total de 32 artículos para la revisión y análisis detallados. 


\section{Análisis de datos}

Se creó una base de datos para registrar y sistematizar la información más relevante de los artículos seleccionados. Los datos que se recogían fueron: título del estudio, autor/es, año y lugar de publicación, muestra del estudio, objetivos, metodología: diseño e instrumentos de recogida de datos y resultados del estudio.

\section{Evaluación de la calidad metodológica}

Se evaluaron los artículos seleccionados de forma independiente. En caso de desacuerdo, se solicitó el juicio de una tercera persona. Para la revisión metodológica de los estudios se evaluaron los artículos con las herramientas JBI Critical Appraisal Tools (Lockwood y Tricco, 2020). En concreto fueron empleados el Checklist for Quasi-Experimental Studies (https://bit.ly/3trIKH4) y el Checklist for Qualitative Research (https://bit.ly/2YFoTWH). Los artículos seleccionados en esta revisión han cumplido satisfactoriamente los criterios señalados.

\section{RESULTADOS}

\section{Análisis descriptivo de la literatura}

Conforme a lo establecido en el proceso estratégico de búsqueda, se registraron en la base de datos ERIC un resultado de 143 artículos (70.09\% con respecto al total) de corte científico ubicados dentro de los objetivos de nuestra investigación, y de 61 artículos (29.90\% con respecto al total) en la base relativa a WOS (períodos de publicación: 2016-2020).

Destaca la prominencia de artículos en relación directa con nuestro objeto de estudio para la franja temporal de 2016 y 2018 respectivamente (69.74\%). Mientras que la base de datos ERIC contiene una mayor cantidad de artículos relacionados con las preguntas del estudio para el período de 2016 a 2019, la base de datos WOS es la que arroja un mayor número de resultados en 2019, siendo 2016 el año en el que figura un menor número de estudios indexados.

En ambas bases de datos, la mayor concentración de estudios sobre la dimensión socioafectiva del clima de aula y sus relaciones con el bienestar emocional de los estudiantes se refieren a las etapas de educación infantil y primaria. Solo el año 2017 (9.37\%) se difunden resultados de investigación sobre esta temática en educación secundaria.

Los estudios descartados correspondieron a aquellos que no cumplían con el objeto de estudio (45.09\%), o que no se centraban en Educación Secundaria 
(12.74\%). Fueron desestimados un $1.47 \%$ de estudios debido a que el idioma en el que habían sido publicados no formaban parte de los criterios de inclusión.

La mayor parte de las investigaciones provienen de Europa (50\%), destacando aquellas publicaciones realizadas en Alemania y España respectivamente. Por otro lado, destacar un número relativamente elevado de estudios ubicados en el continente asiático (25\%), seguido de los Estados Unidos de América (15.6\%). Por último, mencionar la incorporación de investigaciones procedentes de Oceanía $(9.3 \%)$.

Dirigiendo nuestra atención a la metodología empleada, el 37.5\% de los estudios adoptan un enfoque de métodos mixtos. El 34.3\% de las investigaciones son de naturaleza cualitativa, correspondiendo a los estudios de corte cuantitativo un $28.1 \%$. Si atendemos a los instrumentos de recolección de datos en base a la metodología predominante, abunda el uso de cuestionarios y encuestas sucedido por escalas de medición, entrevistas, grupos de discusión, grabaciones en vídeo y observaciones de diversa índole. Las técnicas de análisis estadístico menos empleadas fueron las técnicas de regresión estadística y jerárquica.

De acuerdo con los objetivos perseguidos en esta revisión, cabe destacar que el $62.5 \%$ de los artículos proporcionan evidencias contrastadas sobre la incidencia de un clima de aula afectivo (37.5\%) e inclusivo (25\%) en el bienestar psicosocial de los estudiantes de educación secundaria. También ofrecen evidencias sobre las competencias docentes que lo favorecen cuando se trabaja con adolescentes. De modo más específico, el 37.5\% de los estudios seleccionados demuestran qué competencias docentes promueven un ambiente de aula inclusivo a partir de una adecuada gestión socioafectiva del clima de aula.

\section{Clima afectivo de aula y bienestar adolescente en educación secundaria}

De acuerdo con los resultados obtenidos en cada una de las investigaciones que forman parte de esta revisión sistemática, existen evidencias contrastadas en el impacto de un clima afectivo de aula y el bienestar emocional de los estudiantes de secundaria. El 37.5\% de los artículos objeto de esta revisión ( $\mathrm{N}=11)$ concuerdan en que las relaciones entre calidez afectiva de aula y bienestar emocional de los estudiantes son estrechas. La operativización del constructo "calidez afectiva de aula” se vincula a estas características: ambiente jovial y bullicioso en el que priman las interacciones entre iguales, la comunicación profesorado-grupo de estudiantes, el sentimiento de apoyo percibido por los escolares por parte de sus profesores, el consenso en las normas de funcionamiento del grupo clase, y el apoyo emocional por parte del profesorado (Çengel y Türkoglu, 2016; Hakimzadeh et al., 2016; Bardach et al., 2018; Pöysä et al., 2019). El constructo "bienestar emocional" hace referencia a la realización del propio potencial psicológico resultante de la satisfacción de las necesidades básicas del ser humano. De acuerdo con la teoría de la autodeterminación de Ryan y Deci (2000) 
estas necesidades son: competencia (autoeficacia), autonomía y vinculación (pertenencia).

Asimismo, se evidencia el impacto que un clima afectivo de aula ejerce en la satisfacción vital, establecimiento de metas académicas y vitales, así como también expresión de sentimientos de esperanza y optimismo en los estudiantes (Guess et al., 2013; Quin et al., 2016; Rushton y Kimberley, 2018).

Análogamente, dos estudios revisados demuestran la estrecha relación entre clima afectivo de aula y bienestar emocional de los estudiantes tomando en consideración los niveles de autonomía y participación que perciben en sus clases (Simó et al., 2016; Öqvist y Malmströn, 2016).

Por último, se evidencia que un clima afectivo de aula promueve el desarrollo de un yo positivo. En la práctica se traduce con el desarrollo en los estudiantes de esquemas evaluativos de una manera positiva (autoestima elevada, autoconfianza, motivación intrínseca, percepción de autonomía, sentimientos de autoeficacia personal y uso de estrategias de afrontamiento funcionales) (Cronin et al., 2017, Cremades-Andreu y Lage Gómez, 2018).

\section{Clima inclusivo de aula y bienestar adolescente en educación secundaria}

Un clima inclusivo requiere respetar y prestar atención a las identidades de los estudiantes, combatir la segregación de grupos minoritarios y suscitar sentimientos de pertenencia inter e intragrupal entre los escolares (Brown, 2019). El 25\% de los artículos analizados en esta revisión $(\mathrm{N}=8)$ corroboran cómo un ambiente inclusivo de aula, respetuoso con las singularidades de cada estudiante, genera sentimientos de seguridad y vinculación en el aula, una apreciación positiva de sí mismo, capacidad para gestionar de forma efectiva el entorno y la propia vida, calidad en las relaciones con los demás, propósitos y metas vitales, sentimiento de crecimiento y desarrollo, y sentido de autodeterminación, todos ellos dimensiones asociadas al bienestar psicológico (Ryff y Keyes, 1995).

El estudio de Scharenberg (2016), llevado a cabo en 331 aulas en Alemania, demuestra cómo la segregación y la exclusión en las aulas de educación secundaria incide no solo en el rendimiento académico sino también en el bienestar emocional de los estudiantes. El hecho de que los estudiantes perciban un alto grado de participación en la dinámica de aula incrementa los sentimientos de vinculación y pertenencia al grupo como evidencian las investigaciones de Duchaine et al., (2018), Hanghøj et al., (2018), King y Barry (2019).

Por último, el apoyo social percibido por los estudiantes por parte de sus profesores es lo que activa el sentimiento de vinculación en estudiantes que cursan educación secundaria. Así lo demuestran los estudios que forman parte de esta revisión (Ellery, 2019; Schachner et al., 2019). 
El estilo emocional que exhibe el profesorado en clase explica también cómo se activa el sentimiento de pertenencia en los estudiantes (Eliseev et al., 2019): cuando estos se sentían acogidos y apoyados por sus profesores; cuando sus relaciones con los profesores y sus iguales eran respetuosas, y cuando sentían que estaban aprendiendo.

\section{Competencias docentes y calidad afectiva e inclusiva del clima de aula}

El 37.5\% de los estudios que completan esta revisión de la literatura ( $\mathrm{N}=12)$ focalizan su objeto de estudio en las competencias docentes que promueven un ambiente afectivo e inclusivo de aula con evidencias contrastadas en el bienestar emocional de los estudiantes de educación secundaria. El profesorado juega un papel destacado en la promoción de ambientes de aprendizaje amigables e integradores. Pueden impulsar, o no, la dinámica de aula en pos de la aceptación y el respeto entre los compañeros, la resolución asertiva de conflictos, la negociación, la cooperación y la interdependencia, la participación, la autonomía y el aprecio a la diversidad. Como diseñador del ambiente del aula, el profesorado puede crear o, por el contrario, limitar las oportunidades de los estudiantes para que interactúen socialmente y desarrollen la conciencia social. El profesorado actúa como un referente social y puede modelar actitudes inclusivas, a la vez que movilizar dinámicas afectivas y reacciones emocionales adaptativas en sus estudiantes.

Las investigaciones seleccionadas en esta revisión concluyen que la empatía, la confianza, el respeto a la diversidad, el apoyo emocional, y no solo instructivo, que se brinde a los estudiantes, las oportunidades que se les ofrezcan para participar real y activamente, y no de modo subsidiario, en los asuntos que les competen y afectan, la seguridad psíquica percibida por el estudiante, el reconocimiento, el estímulo y expectativas positivas que se proyecten en los estudiantes resultan claves para liderar un ambiente de aula afectivo e inclusivo (Larmer et al., 2016; Maulana et al., 2016; Chao et al., 2016; Mendenhall et al., 2016; Kulikova et al., 2017; Murphy et al., 2018; Tibu et al., 2018; Gallagher et al., 2019).

Otros estudios evidencian qué estrategias metodológicas resultan más efectivas para dinamizar un clima de aula afectivo e inclusivo y concuerdan que las metodologías dialógicas, cooperativas y participativas redundan en el bienestar emocional de los estudiantes de educación secundaria ya que satisfacen sus necesidades psicológicas básicas (Roczen et al., 2017; Leblanc y SauvaireMaltrana, 2019).

Tan solo dos estudios concluyen subrayando la importancia de incorporar una nueva mirada a la hora de gestionar un ambiente de aprendizaje afectivo desde el enfoque de la inclusión. Señalan que, para ello, se hace necesario adoptar un enfoque centrado en los derechos de la infancia y la adolescencia, situar en el 
centro de la enseñanza el aprender a ser y el bienestar emocional en estas edades (Ghorbani et al., 2018; Sammons et al., 2018).

\section{DISCUSIÓN Y CONCLUSIONES}

La revisión que se presenta se hace eco de una línea de investigación interdisciplinar que en las últimas décadas se ha desarrollado de forma creciente: el estudio del bienestar infantil y adolescente ligado al análisis de los componentes psicosociales y contextos educativos que lo hacen posible.

La presente revisión sistemática se llevó a cabo de acuerdo con dos objetivos. El primero de ellos, consistió en analizar las relaciones existentes entre el ambiente emocional e inclusivo del aula y el bienestar adolescente en educación secundaria. El segundo, se centró en identificar las competencias docentes más relacionadas con la gestión de un ambiente afectivo e inclusivo de aula. Los resultados obtenidos a partir de los 32 estudios seleccionados, son consistentes con los de otros estudios recientes. En relación con los constructos "aula afectiva" y "aula inclusiva" que se han empleado en este estudio, guardan relación con las categorías descriptivas "clima emocional de aula" (CEA) (Alonso-Tapia y Nieto, 2019) y "clima de aula inclusiva" (CAI) (Gistituati et al., 2019). Mientras que la primera (CEA) hace alusión a la relación educativa entre profesorado-estudiantes y las relaciones de los estudiantes con sus iguales y el modo en que a través de dicha relación se satisfacen las necesidades básicas de los escolares (autonomía, competencia, pertenencia), la segunda (CAI) se refiere a las acciones de apoyo que brinda el profesorado a los estudiantes y las que surgen en la dinámica de la relación entre los propios estudiantes, a fin de promover la cohesión y la participación de los estudiantes.

Asimismo, los resultados obtenidos son coherentes con recientes estudios en los que se exploran las percepciones que tienen profesorado y estudiantes de educación secundaria sobre el clima emocional experimentado en las aulas (García-Moya, 2020; Vantieghem et al., 2020). Las valoraciones sobre el clima de respeto y seguridad que viven en las aulas son positivas y compartidas tanto por el profesorado como por los estudiantes. Sin embargo, las mayores diferencias de apreciación se dan en relación con la percepción de la calidez afectiva del aula, siendo los estudiantes quienes declaran percibir un ambiente de aula menos cálido y estimulante desde el punto de vista emocional que el que declara el profesorado (OECD, 2020). Queda por determinar para futuras investigaciones las posibles causas que explican estas diferencias de juicio entre profesorado y estudiantes de educación secundaria.

En relación con las competencias docentes más vinculadas con la gestión de un ambiente afectivo e inclusivo en el aula, los resultados obtenidos en la revisión permiten afirmar que cuando el profesorado establece relaciones de confianza con sus estudiantes, da muestras de afecto y aprecio, así como también 
de reconocimiento a todos los alumnos sin excepción, exhibe una actitud y un comportamiento empático y respetuoso con la diversidad sociocultural, emocional y cognitiva de los estudiantes, resuelve con calidez y firmeza los posibles conflictos que se dan en el aula, está impulsando la autoconfianza, los sentimientos de pertenencia y de valía personal de los estudiantes, así como la socialización en los valores de aprecio y respeto a la diversidad tan importantes en estas edades. Estos gestos profesionales están entrelazados con las competencias emocionales, interpersonales e interculturales de los docentes por lo que se puede concluir que todas ellas requieren una especial atención en la formación inicial y continua del profesorado de educación secundaria.

El estudio realizado presenta varias limitaciones. La primera de ellas es la variabilidad metodológica de los estudios. El hecho de integrar en esta revisión estudios con enfoques metodológicos diferentes puede limitar el alcance de los resultados. La segunda, la franja temporal y la localización de los estudios primarios que se han empleado en la revisión. El hecho de restringir la búsqueda a los cinco últimos años, haber limitado la búsqueda a dos de las principales bases de datos habituales en educación, y no haber incorporado documentos procedentes de literatura gris, limita también los resultados del estudio. Por último, la tercera limitación es de carácter conceptual. La dimensión afectiva en los estudios sobre clima o ambiente de aula suele responder a constructos diferentes, lo que implica que se trabaja desde una categoría multidimensional sin que se advierta una homogeneidad conceptual.

En cualquier caso, los resultados obtenidos corroboran la importancia de la afectividad en las dinámicas interactivas en las aulas y su relación con el bienestar emocional de los adolescentes. Crear un ambiente educativo cálido y respetuoso con la diversidad, incluida la emocional, resultan esenciales para abordar problemáticas relacionadas con el fracaso escolar o el abandono educativo temprano. 


\section{REFERENCIAS BIBLIOGRÁFICAS}

Alonso-Tapia, J., \& Nieto, C. (2019). Classroom emotional climate: Nature, measurement, effects and implications for education. Revista de Psicodidáctica, 24(2). 79-87. https://doi.org/10.1016/j. psicoe.2018.09.002

Archambault, J., \& Chouinard, R. (2016). Vers une gestion éducative de la classe $\left(4^{\mathrm{a}}\right.$ ed.). Gaëtan Morin.

*Bardach, L., Lüftenegger, M., Yanagida, T., Schober, B., \& Spiel, C. (2018). The role of within-class consensus on mastery goal structures in predicting socioemotional outcomes. British Journal of Educational Psychology, 89(2), 239-258. https://doi.org/10.1111/bjep.12237

Berríos-Valenzuela, Ll., \& BuxarraisEstrada, M.R (2013). Educación en valores: análisis sobre las expectativas y los valores de los adolescentes. Educación y Educadores, 16(2), 244-264. https://n9.cl/f3yz0

Brown, C.S. (2019). The importance, and the challenges, to ensuring an inclusive school climate. Educational Psychologist, 54(4), 322-330. https://doi.org/10.1080/0 0461520.2019 .1655646

Bulut Ozsezer, M. S., \& Iflazoglu Saban, A. (2016). An investigation on teacher candidates' perspectives about behaviors positively affecting classroom atmosphere. Eurasian Journal of Educational Research, 16(66), 139-158. https://doi.org/10.14689/ejer.2021.91.5

*Çengel, M., \& Türkoglu, C. (2016). Analysis through hidden curriculum of peer relations in two different classes with positive and negative classroom climates. Educational Sciences: Theory \& Practice, 16(6). 1893-1919. https://doi. org/10.12738/estp.2016.6.0103

Cimpian, M., Maricuţoiu, L. P., \& Ilie, M. D., (2021). Measuring classroom climate: Validation of the CCQ-P for primary, middle school and high school levels, on the Romanian population. Studies in Educational Evaluation, 68. https://doi. org/10.1016/j.stueduc.2021.100976

*Chao, C. N. G., Forlin, C., \& Ho, F. C. (2016). Improving teaching self-efficacy for teachers in inclusive classrooms in Hong Kong. International Journal of Inclusive Education, 20(11), 1142-1154. https://doi.org/10.1080/13603116.2016.1 155663

* Cremades-Andreu, R., \& Lage Gómez, C. (2018). Educación para la paz y violencia escolar a través de un proyecto musical en secundaria. Publicaciones. Facultad de Educación y Humanidades del Campus de Melilla, 48(2), 319-336. https://doi. org/10.30827/publicaciones.v48i2.8347

*Cronin, L. D., Allen, J., Mulvenna, C., \& Russell, P. (2017). An investigation of the relationships between the teaching climate, students' perceived life skills development and well-being within physical education. Physical Education and Sport Pedagogy, 23(2), 181-196. https://doi.org/10.1080/17408989.2017.1 371684

Doyle, W. (1986). Classroom organization and management. En M. C. Wittrock (dir.), Handbook on research on teaching (pp. 392-431). MacMillan.

*Duchaine, E., Jolivette, K., Fredrick, L., \& Alberto, P.L. (2018). Increase engagement and achievement with response cards: Science and Mathematics inclusion classes. Learning Disabilities: A Contemporary Journal, 16(2), 157-176. https://n9.cl/88svk

*Ellery, T. (2019). Belonging as a pathway to inclusive: an inquiry into supporting inclusive practice in secondary schools. Kairaranga, 20(2), 52-62. https://n9.cl/ $\mathrm{n} 8 \mathrm{xh}$

* Eliseev, V., Eliseeva, I., Dedyaeva, U., Korobova, M., \& Romanova, U. (2019). The impact of the educator's 
emotional orientation and personal qualities on the process of gaining authority among socially maladjusted adolescents. Amazonia Investiga, 8(23), 487-496. https://bit.ly/357jfPF

European Commission (2019). PISA 2018 and the EU. Striving for social fairness through education. Directorate-General for Education, Youth, Sport and Culture.

European Commission/EACEA/Euridyce (2019). Integrating students for migrant backgrounds into schools in Europe: National policies and measures. Eurydice Report. Publications Office of the European Union. https://n9.cl/ee3h

Fakunle, F. E., \& Ale, M. V. (2018). School climate as determinant of students' academic performance in public secondary schools in Ekiti State, Nigeria. African Educational Research Journal, 6(4), 236-239. https://doi. org/10.30918/aerj.64.18.055

Fraser, B.J. (1998). Classroom environment instruments: Development, validity and applications. Learning Environments Research, 1(1), 7-34. https://doi. org/10.1023/A:1009932514731

García-Moya, I. (2020). The importance of connectedness in studentteacher relationships. Palgrave MacMillan. https://doi.org/10.1007/9783-030-43446-5

Ghaith, G. (2003). The relationship between forms of instruction, achievement and perceptions of classroom climate. Educational Research, 45(1), 83-93. https://doi. org/10.1080/0013188032000086145

*Ghorbani, S., Mirshah Jafari, S. E., \& Sharifian, F. (2018). Learning to be: teachers' competences and practical solutions: A step towards sustainable development. Journal of Teacher Education for Sustainability, 20(1), 2045. https://doi.org/10.2478/jtes-20180002
Gizir, S. (2019). The sense of classroom belonging among pre-service teachers: Testing a theoretical model. European Journal of Educational Research, 8(1), 8797. https://doi.org/10.12973/eu-jer.8.1.87

Gouch, D., Oliver, S., \& Thomas, J. (2017). An Introduction to Systematics Reviews (2 ${ }^{\mathrm{a}}$ ed.) Sage.

*Guess, P. E., \& McCane-Bowling, S. J. (2013). Teacher Support and Life Satisfaction. Education and Urban Society, 48(1), 30-47. https://doi. org/10.1177/0013124513514604

*Hakimzadeh, R., Besharat, M.-A., Khaleghinezhad, S. A., \& Ghorban Jahromi, R. (2016). Peers' perceived support, student engagement in academic activities and life satisfaction: A structural equation modeling approach. School Psychology International, 37(3), 240-254. https://doi. org/10.1177/0143034316630020

Hanghøj, T., Lieberoth, A., \& Misfeldt, M. (2018). Can cooperative video games encourage social and motivational inclusion of at-risk students? British Journal of Educational Technology, 49(4), 775-799.

Hutton, B., Salanti, G., Caldwell, D. M., Chaimani, A., Schmid, C. H., Cameron, C., Moher, D., Loannidis, J., Straus, S., Thorlund, K., Jansen, J., Mulrow, C., Catalá-López, C., Gøtzsche, P., Dickersin, K., Boutron, I., Altman, D., \& Moher, D. (2015). The PRISMA extension statement for reporting of systematic reviews incorporating network meta-analyses of health care interventions: Checklist and explanations. Annals of Internal Medicine, 162(11), 777-784. https://doi. org/10.7326/M14-2385

Kalin, J., Peklaj, C., Pečjak, S., Levpušček, M. P., \& Zuljan, M. V. (2017). Elementary and secondary school students' perceptions of teachers' classroom management competencies. Ceps, 7(4), 37-62. https://n9.cl/ldm4 
*King, N., \& Ryan, B.J (2019). The impact of social inclusion on the social development of students with a general learning difficulty in postprimary education in Ireland, Education Research International, 2019, 1-7. https://doi. org/10.1155/2019/6327567

Kulikova, T.I., \& Maliy, D.V. (2017). Professional and personal qualities of the teacher in the context of the psychological safety of educational environment. European Journal of Contemporary Education, 6(4), 715-722. https://bit.ly/2H5L56u

*Kulikova, T.I., Shalaginova, K.S. \& Cherkasova, S.A. (2017). The polyethnic competence of class teacher as a resource for ensuring the psychological security of pupils in a polycultural educational environment. European Journal of Contemporary Education, 6(3), 557-564. https://doi.org/10.13187/ejced.2017.3.557

Kutsyuruba, B., Klinger, D. A., \& Hussain, A. (2015). Relationships among school climate, school safety, and student achievement and well-being: a review of the literature. Review of Education, 3(2), 103-135. https://doi.org/10.1002/ rev3.3043

*Gallagher, E. K., Dever, B. V., Hochbein, C., \& DuPaul, G. J. (2019). Teacher caring as a protective factor: The effects of behavioral/emotional risk and teacher caring on office disciplinary referrals in middle school. School Mental Health, 11(4), 754-765. https://doi.org/10.1007/ s12310-019-09318-0

*Larmer, W., Baker, C., \& Gentry, J. (2016). A model for online support in classroom management: Perceptions of beginning teachers. Administrative Issues Journal: Education, Practice, and Research, 6(1). 22-37. https://doi.org/10.5929/2016.6.1.3

*Leblanc, S., \& Sauvaire-Maltrana, C. (2019). Understanding the "atypical" activity of teachers: example of collaborative pedagogical format in secondary school. @ctivités, 6(1), 1-34. https://doi.org/10.4000/activites.3901

Lee, V. E., \& Smith, J. B. (1999). Social support and achievement for young adolescents in Chicago: The role of school academic press. American Educational Research Journal, 36(4), 907-945. https:// doi.org/10.3102/00028312036004907

Lockwood, C., \& Tricco, A.C. (2020). Preparing scoping reviews for publication using methodological guides and reporting standards. Nursing \& Health Sciences, 22, 1-4. https://doi. org/10.1111/nhs.12673

*Maulana, R., Helms-Lorenz, M., Irnidayanti, Y., \& Van de Grift, W. (2016). Autonomous motivation in the Indonesian classroom: relationship with teacher support through the lens of SelfDetermination Theory. The Asia-Pacific Education Researcher, 25(3), 441-451. https://doi.org/10.1007/s40299-0160282-5

*Mendenhall, M., Bartlett, L., \& GhaffarKucher, A. (2016). "If you need help, they are always there for us": Education for refugees in an international high school in NYC. The Urban Review, 49(1), 1-25. https://doi.org/10.1007/s11256-0160379-4

Moher, D., Liberati, A., Tetzlaff, J., \& Altman, D.J. (2009). Preferred reporting items for systematic reviews and metaanalyses: The PRISMA statement. PLoS Med. 6(7). https://doi.org/10.1371/ journal.pmed.1000097

Moos, R.H. \& Trickett, E. (1974). Classroom Environment Scale. APA PsycTests. https://doi.apa.org/doi/10.1037/t06449000

*Murphy, H. R., Tubritt, J., \& O’Higgins Norman, J. (2018). The role of empathy in preparing teachers to tackle bullying. Journal of New Approaches in Educational Research, 7(1), 17-23. https://doi.org/10.7821/naer.2018.1.261 
Naghsh Daemi, M., Tahriri, A., \& Mahdavi Zafarghandi, A. (2017). The relationship between classroom environment and EFL learners' academic selfefficacy. International Journal of Education and Literacy Studies, 5(4), 16. https://doi.org/10.7575/aiac. ijels.v.5n.4p.16

Newland, L. A., De Cino, D. A., Mourlam, D. J., \& Strouse, G. A. (2019). School climate, emotions, and relationships: Children's experiences of well-being in the Midwestern U.S. International Journal of Emotional Education, 11(1), 67-83.

Newman, M., \& Gough, D., (2020). Methodological considerations. En O. Zawacki-Ritcher, M. Kerres, S. Bendenlier, M. Bond y K. Buntins, Systematic reviews in educational research. Methodology, perspectives and application (pp. 3-22). Springer. https://doi.org/10.1007/978-3658-27602-7

Nisar, N., Mahmood, M. K., \& Dogar, A. H., (2017). Determinants of students' academic achievement at secondary school level. Bulletin of Education and Research, 39(1), 145-158. https://bit. ly/3nN7iHr

OECD (2020). Global teaching Iinsights. A video study of teaching. OECD Publishing. https://doi.org/10.1787/20d6f36b-en

*Olmos Rueda, P., Sanahuja Gavaldà, J., \& Mas Torelló, Ó. (2018). El alumnado de educación secundaria obligatoria ante la inclusión educativa y la docencia compartida. REOP - Revista Española de Orientación y Psicopedagogía, 29(3), 8-24. https://doi.org/10.5944/reop.vol.29. num.3.2018.23318

*Öqvist, A., \& Malmström, M. (2016). Teachers' leadership: a maker or a breaker of students' educational motivation. School Leadership \& Management, 36(4), 365-380. https://doi. org/10.1080/13632434.2016.1247039
*Pöysä, S., Vasalampi, K., Muotka, J., Lerkkanen, M., Poikkeus, A., \& Nurmi, J. (2018). Teacher-student interaction and lower secondary school students' situational engagement. British Journal of Educational Psychology, 89(2), 374392. https://doi.org/10.1111/bjep.12244

*Quin, D., Hemphill, S. A., \& Heerde, J. A. (2017). Associations between teaching quality and secondary students' behavioral, emotional, and cognitive engagement in school. Social Psychology of Education, 20(4), 807-829. https://doi. org/10.1007/s11218-017-9401-2

Romero-Pérez, C., Torres-Savater, $\mathrm{M}^{\mathrm{a}} \mathrm{C}$., \& Traver Martí, J.A. (2019). Formar para la relación educativa desde la profesión docente. En J. Vera Vila (Coord.), Formar para transformar. Cambio social y profesiones educativas (pp. 217-240). GEU Editorial. https://bit.ly/3157vw7

*Roczen, N., Abs, H. J., \& Filsecker, M. (2017). How school influences adolescents' conflict styles. Journal of Peace Education, 14(3), 325-346. https:// doi.org/10.1080/17400201.2017.1345728

*Rushton, C., \& Wilson, K. (2018). Understanding the work of FLOs through a recovery framework lens. International Journal of Inclusive Education, 24(1), 1-14. https://doi.org/10.1080/13603116. 2018.1448122

Ryan, R. M., \& Deci, E. L. (2000). Self-determination theory and the facilitation of intrinsic motivation, social development and well-being. American Psychologist, 55(1), 68-78. https://doi. org/10.1037/0003-066X.55.1.68

Ryff, C. D., \& Keyes, C. L. M. (1995). The structure of psychological well-being revisited. Journal of Personality and Social Psychology, 69(4), 719-727. https:// doi.org/10.1037/0022-3514.69.4.719

*Sammons, P., Kington, A., Lindorff, A., \& Ortega, L. (2018). 'It ain't (only) what you do, it's the way that you do it': A mixed method approach to the study of 
inspiring teachers. Review of Education, 6(3), 303-356. https://doi.org/10.1002/ rev3.3141

Sánchez-Meca, J. S. (2010). Cómo realizar una revisión sistemática y un metaanálisis. Aula abierta, 38(2), 53-64.

*Scharenberg, K. (2016). The interplay of social and ethnic classroom composition, tracking, and gender on students' school satisfaction. Journal of Cognitive Education and Psychology, 15(2), 320346. https://doi.org/10.1891/19458959.15.2.320

*Schachner, M. K., Schwarzenthal, M., van de Vijver, F. J. R., \& Noack, P. (2019). How all students can belong and achieve: Effects of the cultural diversity climate amongst students of immigrant and nonimmigrant background in Germany. Journal of Educational Psychology, 111(4), 703-716. https://doi.org/10.1037/ edu0000303

Shindler, J., Jones, A., Williams, A. D., Taylor, C., \& Cardenas, H. (2016). The school climate-student achievement connection: if we want achievement gains, we need to begin by improving the climate. Journal of School Administration Research and Development, 1(1), 9-16.

Sherblom, S. A., Marshall, J. C., \& Sherblom, J. C. (2006). The relationship between school climate and math and reading achievement. Journal of Research in Character Education, 4(1), 19-31.

Sieberer-Nagler, K. (2015). Effective classroom-management and positive teaching. English Language Teaching, 9(1), 163. https://doi. org/10.5539/elt.v9n1p163

*Simó, N., Parareda, A., \& Domingo, L. (2016). Towards a democratic school: The experience of secondary school pupils. Improving Schools, 19(3), 181-196. https://doi. org/10.1177/1365480216631080
Thapa, A. (2013). School climate and research. En T. Dary y T. Pickeral (Ed.), School climate practices for implementation and sustainability. A school climate practice brief, 1 (pp.13-16). National School Climate Center. https:// bit.ly/3uiPs2i

*Tibu, S. L., \& Horga, I. (2018). Empathic and supportive teachers, key to quality and efficiency in education. Revista Romaneasca Pentru Educatie Multidimensionala, 10(4), 166-181. https://doi.org/10.18662/rrem/80

Vantieghem, W., Roose, I., Gheyssens, E., Griful-Freixenet, J., Keppens, K., Vanderlinde, R., \& Van Avermaet, P. (2020). Professional vision of inclusive classrooms: A validation of teachers' reasoning on differentiated instruction and teacher-student interactions. Studies in Educational Evaluation, 67. https:// doi.org/10.1016/j.stueduc.2020.100912

Vera Vila, J. (Coord.) (2019). Formar para transformar. Cambio social y profesiones educativas. GEU Editorial. https://bit. ly/3157vw7

Viau, R. (2009). La motivation en contexte scolaire ( $2^{\mathrm{a}}$ ed.). De Boeck.

Voight, A., \& Hanson, T. (2017). How are middle school climate and academic performance related across schools and over time? National Centre for Education Evaluation and Regional Assistance. https://n9.cl/9u4mt

Whitlock, J. L. (2006). Youth perceptions of life in school: Contextual correlates of school connectedness in adolescence. Applied Developmental Science, 10, 13-29. https://doi.org/10.1207/ s1532480xads1001_2

Yeager, D. S. (2017). Social and emotional learning programs for adolescents. Future of Children, 27(1), 7394. https://doi.org/10.1353/foc.2017.0004 


\section{ANEXOS}

Tabla 1

Búsqueda en la base de datos Education Recources Informacion Center (ERIC)

\begin{tabular}{|c|c|c|}
\hline Búsqueda & Términos de búsqueda & Resultados \\
\hline$\# 1$ & Educational Environment AND Inclusion & 95 \\
\hline \#2 & Educational Environment AND School Climate & 212 \\
\hline$\# 3$ & $\begin{array}{l}\text { (School Climate OR Educational Environment) } \\
\text { AND Inclusión }\end{array}$ & 129 \\
\hline$\# 4$ & Inclusion AND Risk Students & 35 \\
\hline \#5 & (Inclusion AND Well Being) NOT Violence & 108 \\
\hline \#6 & (Inclusion AND Well Being) NOT School Security & 10 \\
\hline$\# 7$ & $\begin{array}{l}\text { (Student Satisfaction AND Student Welfare) NOT } \\
\text { Teacher Welfare }\end{array}$ & 215 \\
\hline$\# 8$ & Organizational Climate & 56 \\
\hline$\# 9$ & Class Organization & 38 \\
\hline$\# 10$ & $\begin{array}{l}\text { (Class Organization OR Organizational Climate) } \\
\text { AND Teacher Competencies }\end{array}$ & 1746 \\
\hline$\# 11$ & Mental Health AND Student Welfare & 15 \\
\hline$\# 12$ & $\begin{array}{l}\text { (Teaching conditions AND Teacher Student Rela- } \\
\text { tionship) AND Teacher Competencies }\end{array}$ & 36 \\
\hline$\# 13$ & $\begin{array}{l}\text { Psychosocial Development AND Educational En- } \\
\text { vironment }\end{array}$ & 5 \\
\hline \#14 & $\begin{array}{l}\text { Educational Environment OR Psychosocial Deve- } \\
\text { lopment AND Well Being }\end{array}$ & 500 \\
\hline \#15 & $\begin{array}{l}\text { (Psychosocial Development AND Educational } \\
\text { Environment) AND Well Being }\end{array}$ & 105 \\
\hline$\# 16$ & Psychosocial Development & 31 \\
\hline$\# 17$ & Psychosocial Development AND Well Being & 4 \\
\hline$\# 18$ & $\begin{array}{l}\text { (Teacher Competencies AND Teacher Influence) } \\
\text { NOT Academic Failure }\end{array}$ & 562 \\
\hline \#19 & $\begin{array}{l}\text { (Educational environment OR Mental Health) } \\
\text { AND Psychosocial Development }\end{array}$ & 63 \\
\hline \#20 & $\begin{array}{l}\text { (Student Welfare AND Teacher Student Rela- } \\
\text { tionship) NOT Student College Relationship }\end{array}$ & 709 \\
\hline$\# 21$ & Inclusion AND Well Being & 29 \\
\hline \#22 & Teacher Competencies AND Teacher Influence & 64 \\
\hline
\end{tabular}


Tabla 2

Búsqueda en la base de datos Web of Science (WOS)

\begin{tabular}{|c|c|c|c|c|c|}
\hline Búsqueda & $\begin{array}{l}\text { Términos de } \\
\text { búsqueda }\end{array}$ & $\underset{1}{\text { Resultado }}$ & $\begin{array}{c}\text { Resultado } \\
2: \\
\text { secondary } \\
\text { school }\end{array}$ & $\begin{array}{l}\text { Resultado } \\
\text { 3: lower } \\
\text { secondary } \\
\text { general } \\
\text { education }\end{array}$ & $\begin{array}{l}\text { Resultado } \\
\text { 4: upper } \\
\text { secondary } \\
\text { education }\end{array}$ \\
\hline$\# 1$ & $\begin{array}{l}\text { Educational } \\
\text { Environment AND } \\
\text { Inclusion }\end{array}$ & 409 & 31 & 2 & - \\
\hline$\# 2$ & $\begin{array}{l}\text { Educational } \\
\text { Environment AND } \\
\text { School Climate }\end{array}$ & 183 & 18 & 1 & 1 \\
\hline$\# 3$ & $\begin{array}{l}\text { (School Climate } \\
\text { OR Educational } \\
\text { Environment) AND } \\
\text { Inclusión }\end{array}$ & 7 & - & - & - \\
\hline$\# 4$ & $\begin{array}{l}\text { Inclusion AND Risk } \\
\text { Students }\end{array}$ & 362 & 22 & 1 & 4 \\
\hline$\# 5$ & $\begin{array}{l}\text { (Inclusion AND } \\
\text { Well Being) NOT } \\
\text { Violence }\end{array}$ & 1.277 & 36 & 2 & 3 \\
\hline$\# 6$ & $\begin{array}{l}\text { (Inclusion AND } \\
\text { Well Being) NOT } \\
\text { School Security }\end{array}$ & 1.317 & 37 & 2 & 3 \\
\hline$\# 7$ & $\begin{array}{l}\text { (Student } \\
\text { Satisfaction AND } \\
\text { Student Welfare) } \\
\text { NOT Teacher } \\
\text { Welfare }\end{array}$ & 38 & 2 & - & - \\
\hline$\# 8$ & $\begin{array}{l}\text { Organizational } \\
\text { Climate }\end{array}$ & 2.761 & 19 & 1 & 2 \\
\hline$\# 9$ & Class Organization & 2.673 & 67 & 1 & 6 \\
\hline$\# 10$ & $\begin{array}{l}\text { (Class Organization } \\
\text { OR Organizational } \\
\text { Climate) } \\
\text { AND Teacher } \\
\text { Competencies }\end{array}$ & 3 & - & - & - \\
\hline$\# 11$ & $\begin{array}{l}\text { Mental Health AND } \\
\text { Student Welfare }\end{array}$ & 72 & 4 & 1 & - \\
\hline
\end{tabular}




\begin{tabular}{|c|c|c|c|c|c|}
\hline Búsqueda & $\begin{array}{l}\text { Términos de } \\
\text { búsqueda }\end{array}$ & $\underset{1}{\text { Resultado }}$ & $\begin{array}{c}\text { Resultado } \\
\text { 2: } \\
\text { secondary } \\
\text { school }\end{array}$ & $\begin{array}{l}\text { Resultado } \\
\text { 3: lower } \\
\text { secondary } \\
\text { general } \\
\text { education }\end{array}$ & $\begin{array}{l}\text { Resultado } \\
\text { 4: upper } \\
\text { secondary } \\
\text { education }\end{array}$ \\
\hline$\# 12$ & $\begin{array}{l}\text { (Teaching } \\
\text { conditions AND } \\
\text { Teacher Student } \\
\text { Relationship) } \\
\text { AND Teacher } \\
\text { Competencies }\end{array}$ & 3 & - & - & - \\
\hline$\# 13$ & $\begin{array}{l}\text { Psychosocial } \\
\text { Development } \\
\text { AND Educational } \\
\text { Environment }\end{array}$ & 39 & 1 & - & - \\
\hline$\# 14$ & $\begin{array}{l}\text { Educational } \\
\text { Environment } \\
\text { OR Psychosocial } \\
\text { Development AND } \\
\text { Well Being }\end{array}$ & 833 & 32 & - & 1 \\
\hline \#15 & $\begin{array}{l}\text { (Psychosocial } \\
\text { Development } \\
\text { AND Educational } \\
\text { Environment) AND } \\
\text { Well Being }\end{array}$ & 834 & 32 & - & 1 \\
\hline$\# 16$ & $\begin{array}{l}\text { Psychosocial } \\
\text { Development }\end{array}$ & 3.487 & 45 & - & 1 \\
\hline$\# 17$ & $\begin{array}{l}\text { Psychosocial } \\
\text { Development AND } \\
\text { Well Being }\end{array}$ & 415 & 14 & - & - \\
\hline$\# 18$ & $\begin{array}{l}\text { (Teacher } \\
\text { Competencies AND } \\
\text { Teacher Influence) } \\
\text { NOT Academic } \\
\text { Failure }\end{array}$ & 214 & 22 & - & 2 \\
\hline$\# 19$ & $\begin{array}{l}\text { (Educational } \\
\text { environment OR } \\
\text { Mental Health) } \\
\text { AND Psychosocial } \\
\text { Development }\end{array}$ & 859 & 10 & - & - \\
\hline
\end{tabular}




\begin{tabular}{|c|c|c|c|c|c|}
\hline Búsqueda & $\begin{array}{l}\text { Términos de } \\
\text { búsqueda }\end{array}$ & $\underset{1}{\text { Resultado }}$ & $\begin{array}{c}\text { Resultado } \\
2: \\
\text { secondary } \\
\text { school }\end{array}$ & $\begin{array}{l}\text { Resultado } \\
\text { 3: lower } \\
\text { secondary } \\
\text { general } \\
\text { education }\end{array}$ & $\begin{array}{l}\text { Resultado } \\
\text { 4: upper } \\
\text { secondary } \\
\text { education }\end{array}$ \\
\hline$\# 20$ & $\begin{array}{l}\text { (Student } \\
\text { Welfare AND } \\
\text { Teacher Student } \\
\text { Relationship) NOT } \\
\text { Student College } \\
\text { Relationship }\end{array}$ & 14 & 2 & - & - \\
\hline$\# 21$ & $\begin{array}{l}\text { Inclusion AND Well } \\
\text { Being }\end{array}$ & 1.326 & 47 & 2 & 3 \\
\hline \#22 & $\begin{array}{l}\text { Teacher } \\
\text { Competencies AND } \\
\text { Teacher Influence }\end{array}$ & 216 & 22 & - & 2 \\
\hline
\end{tabular}




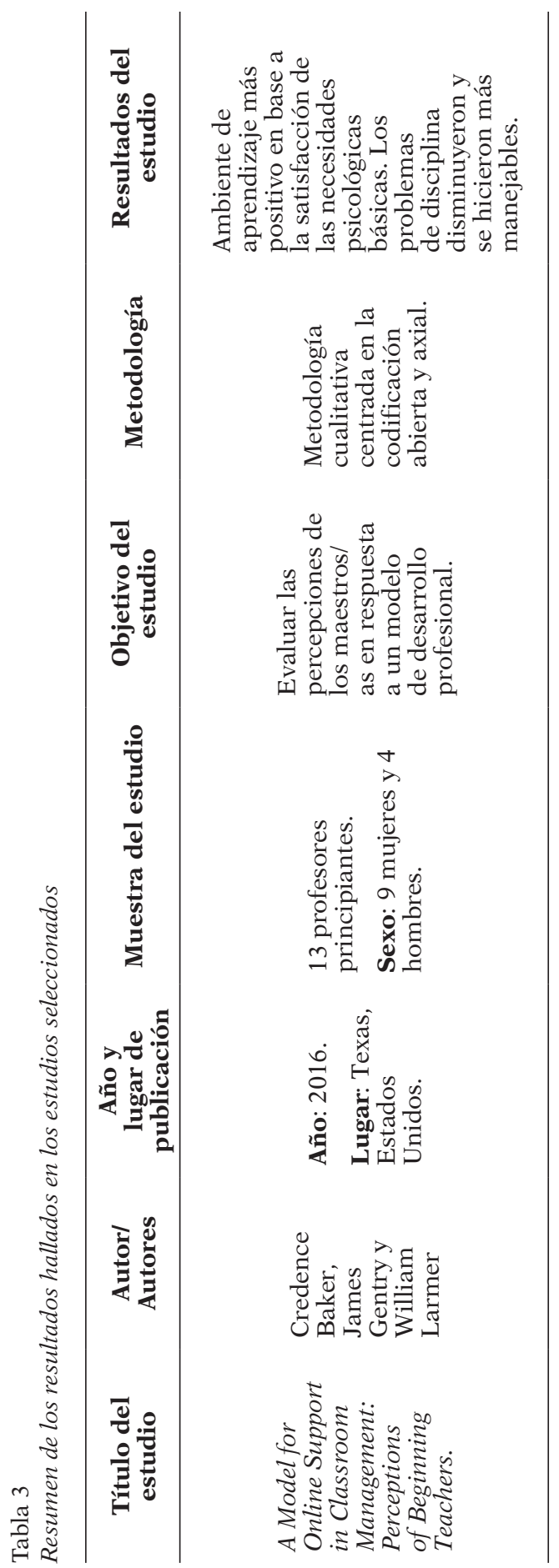

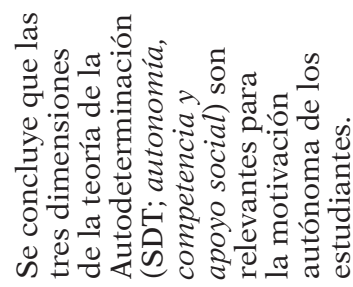
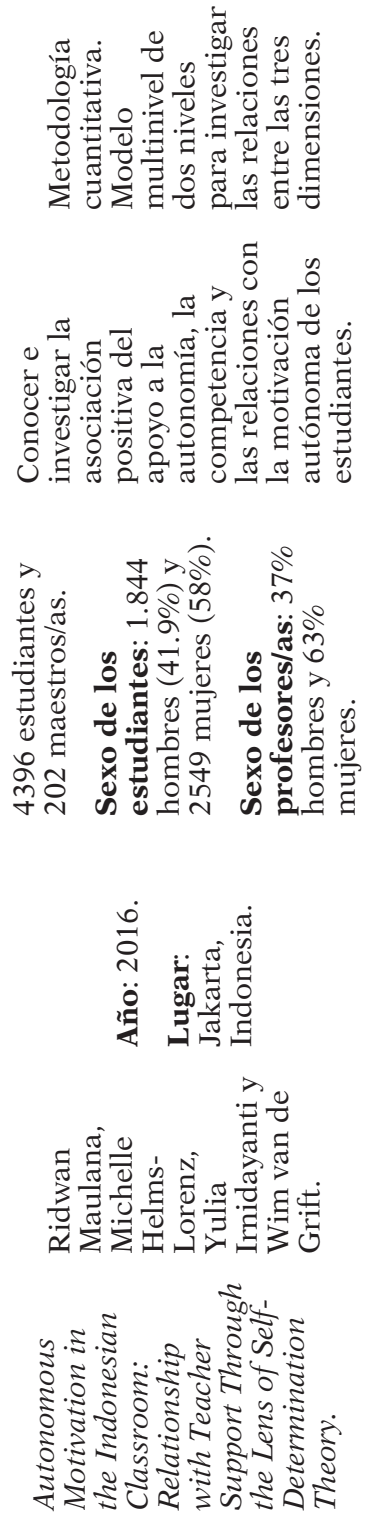


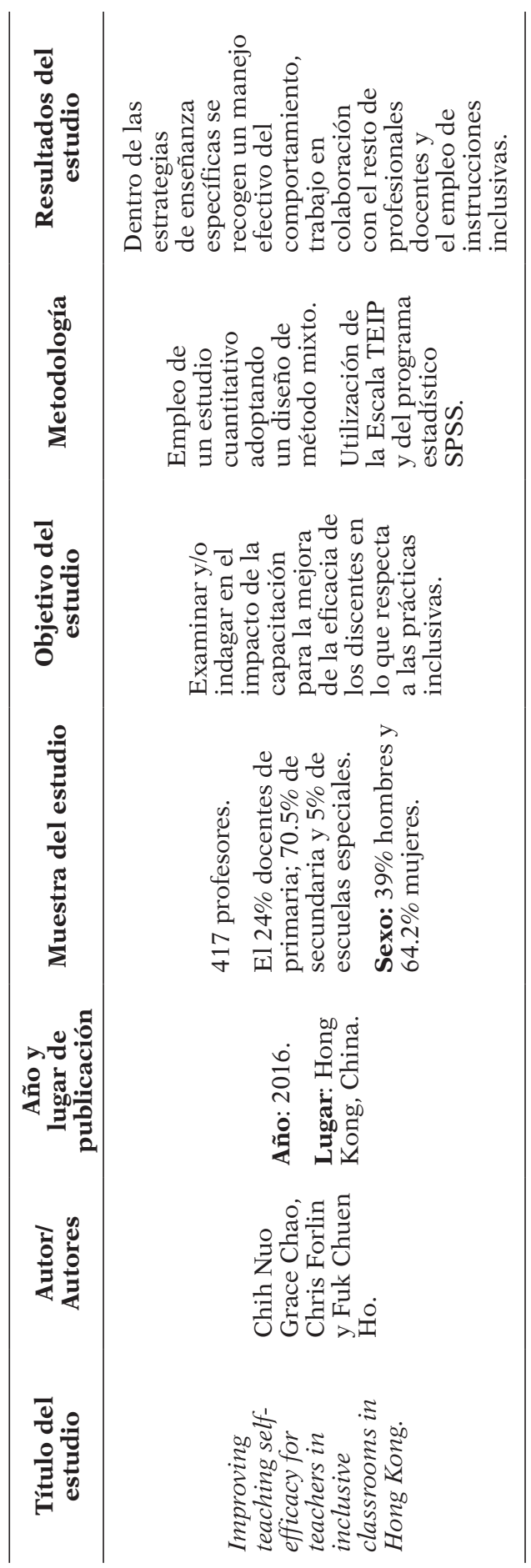

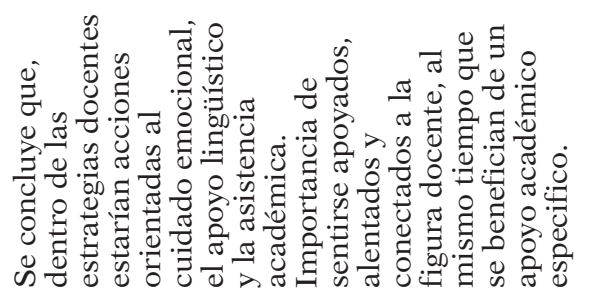
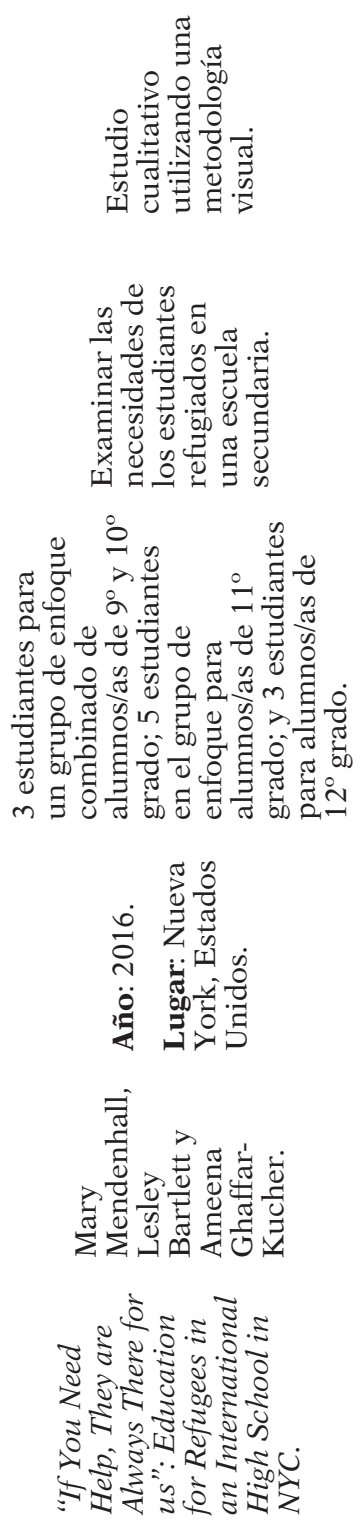


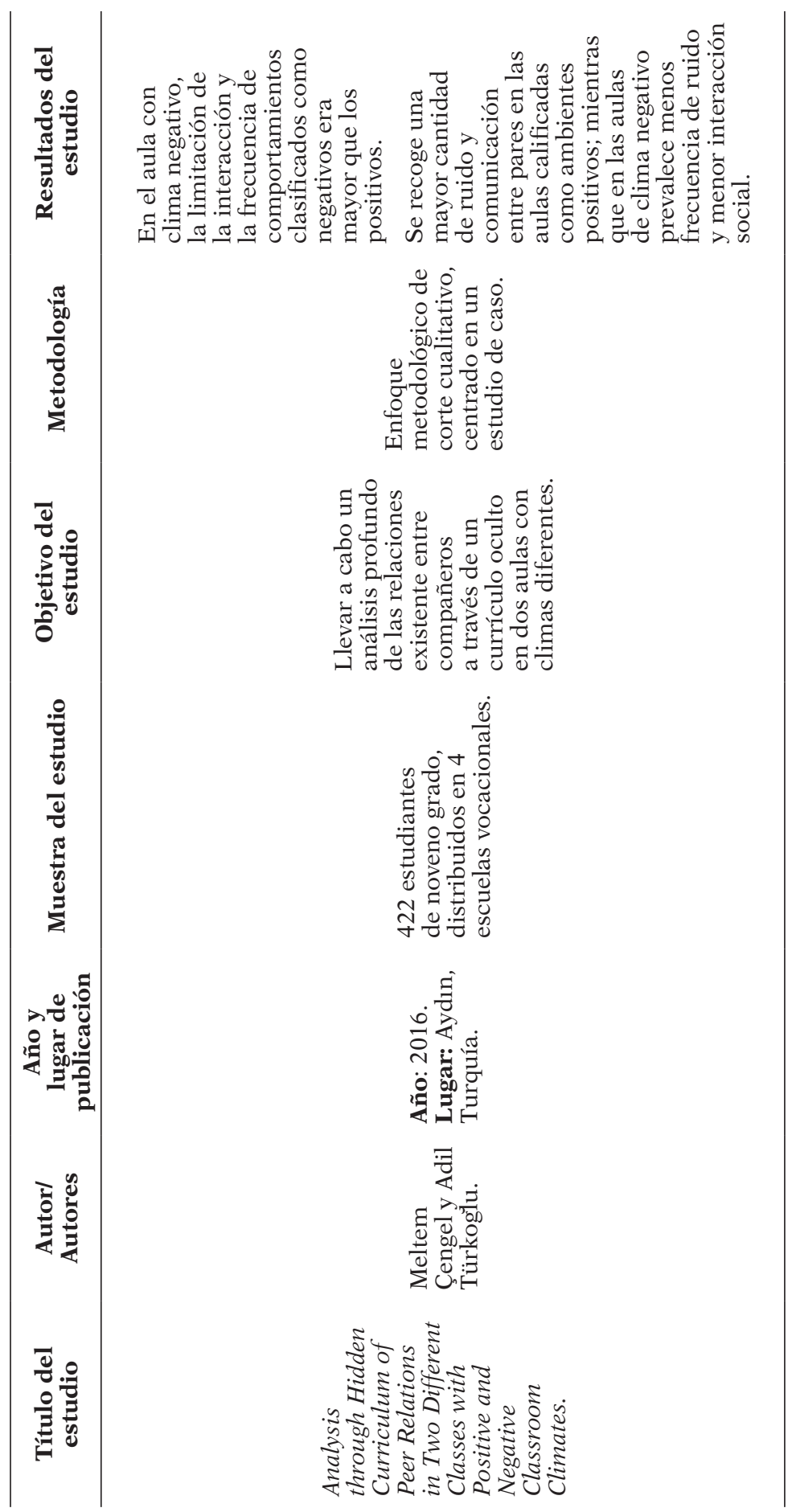




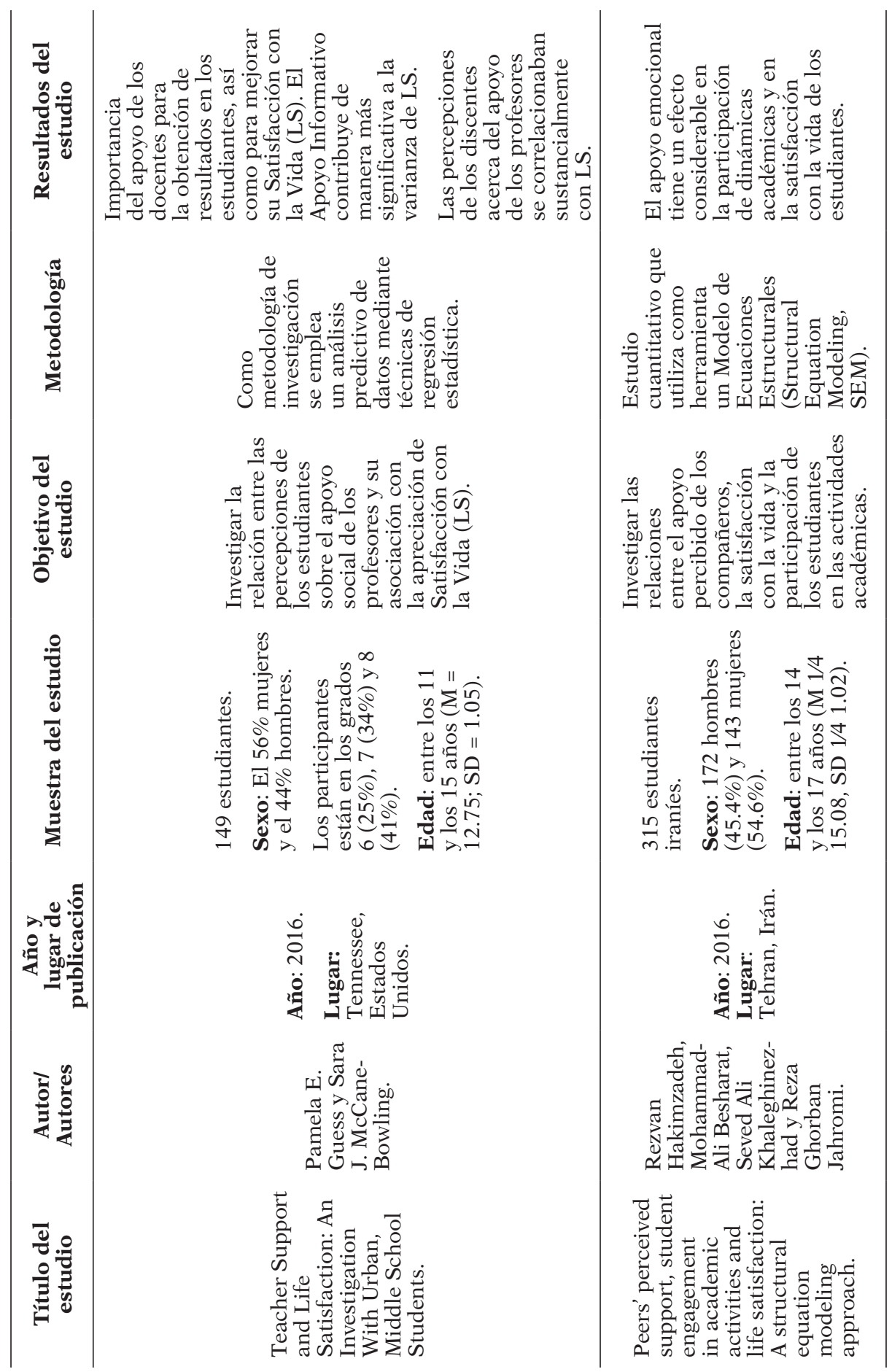




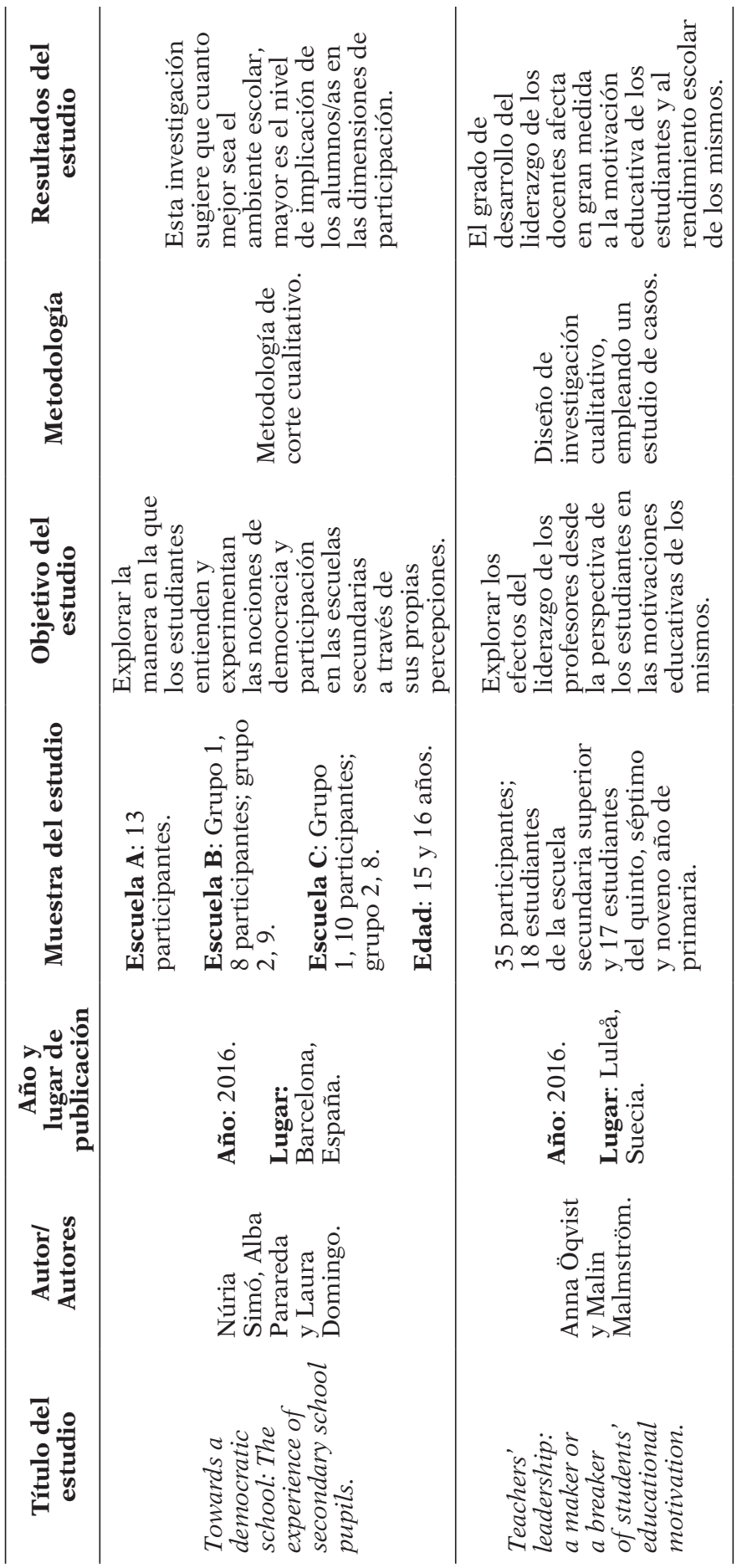




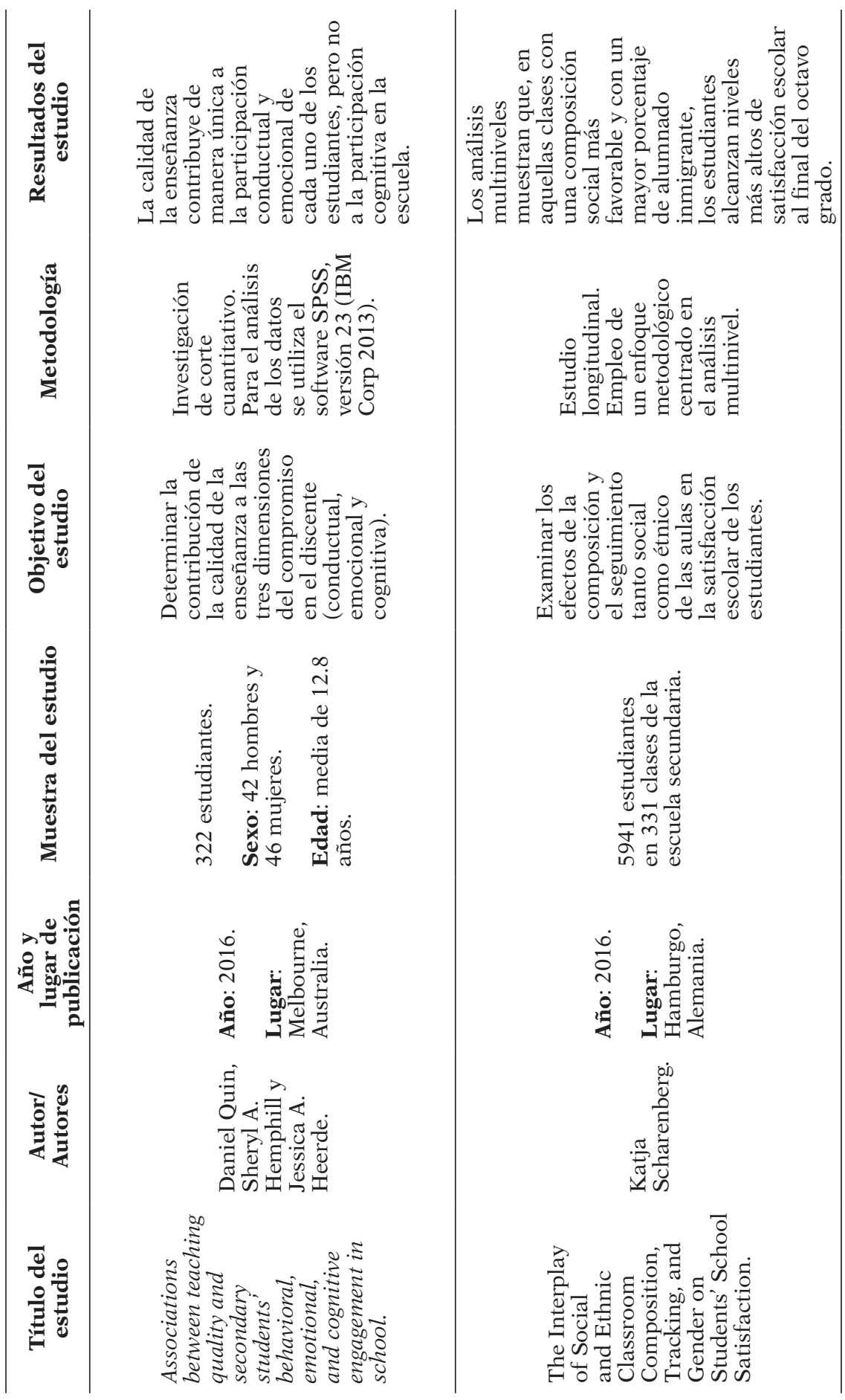




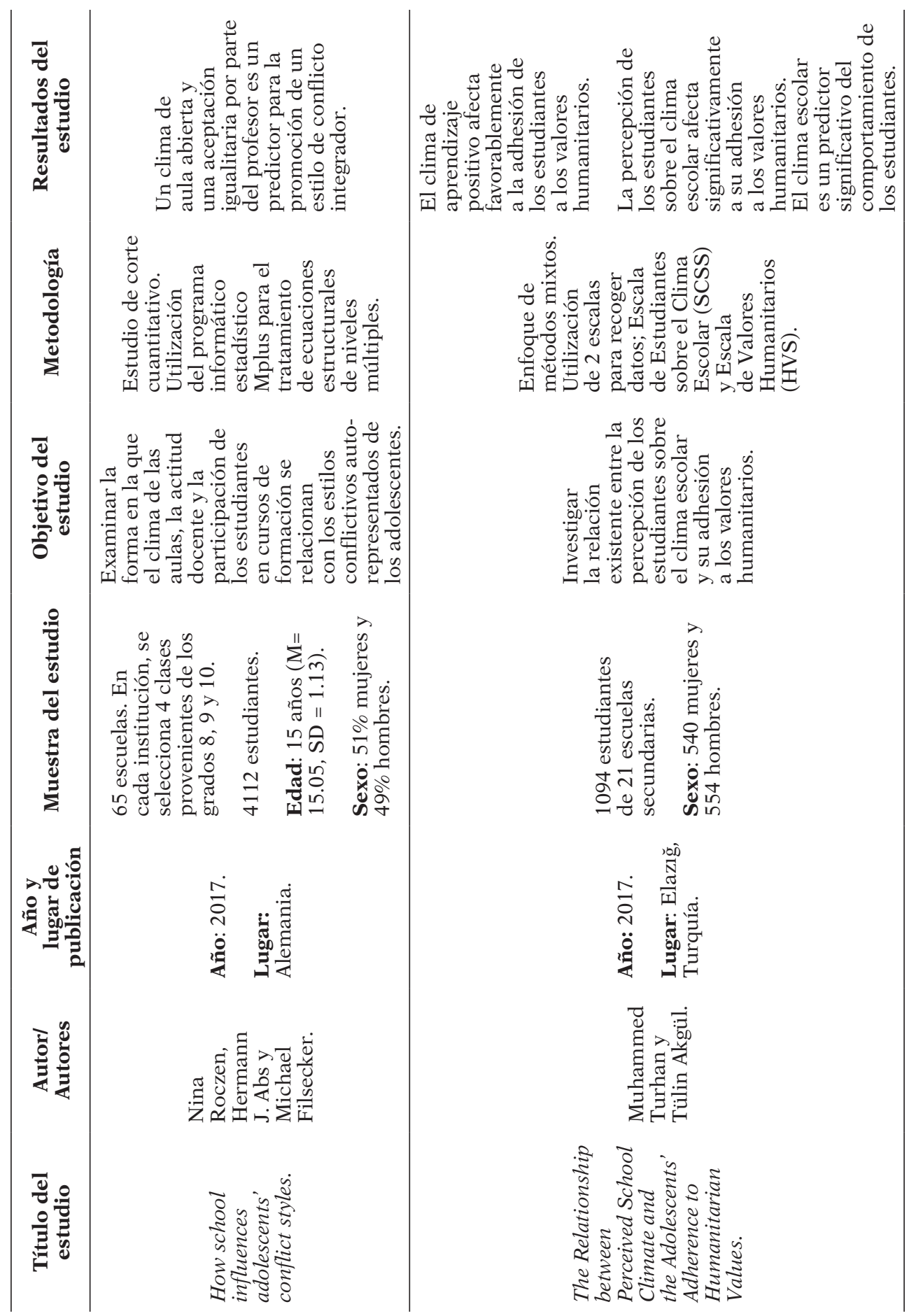




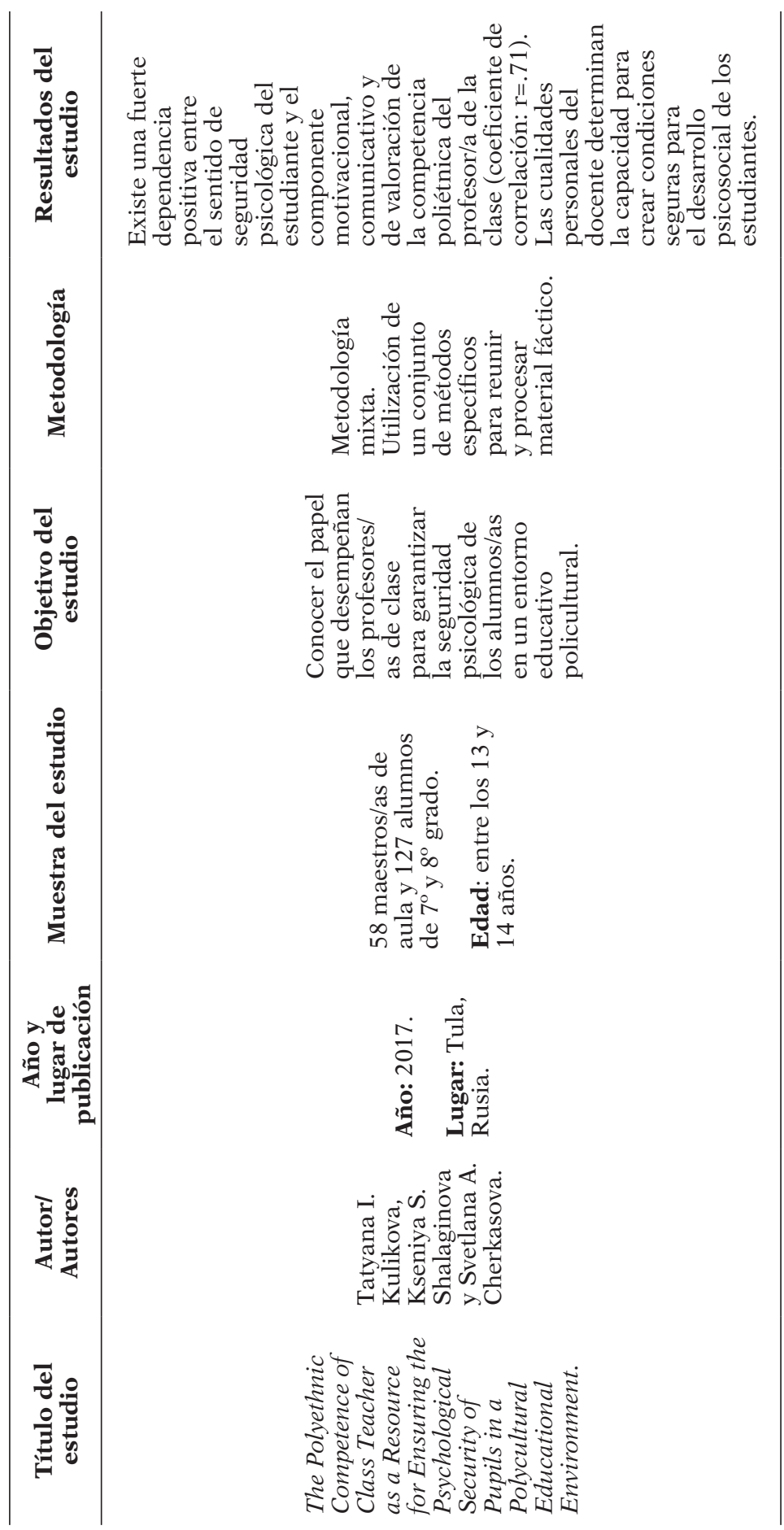




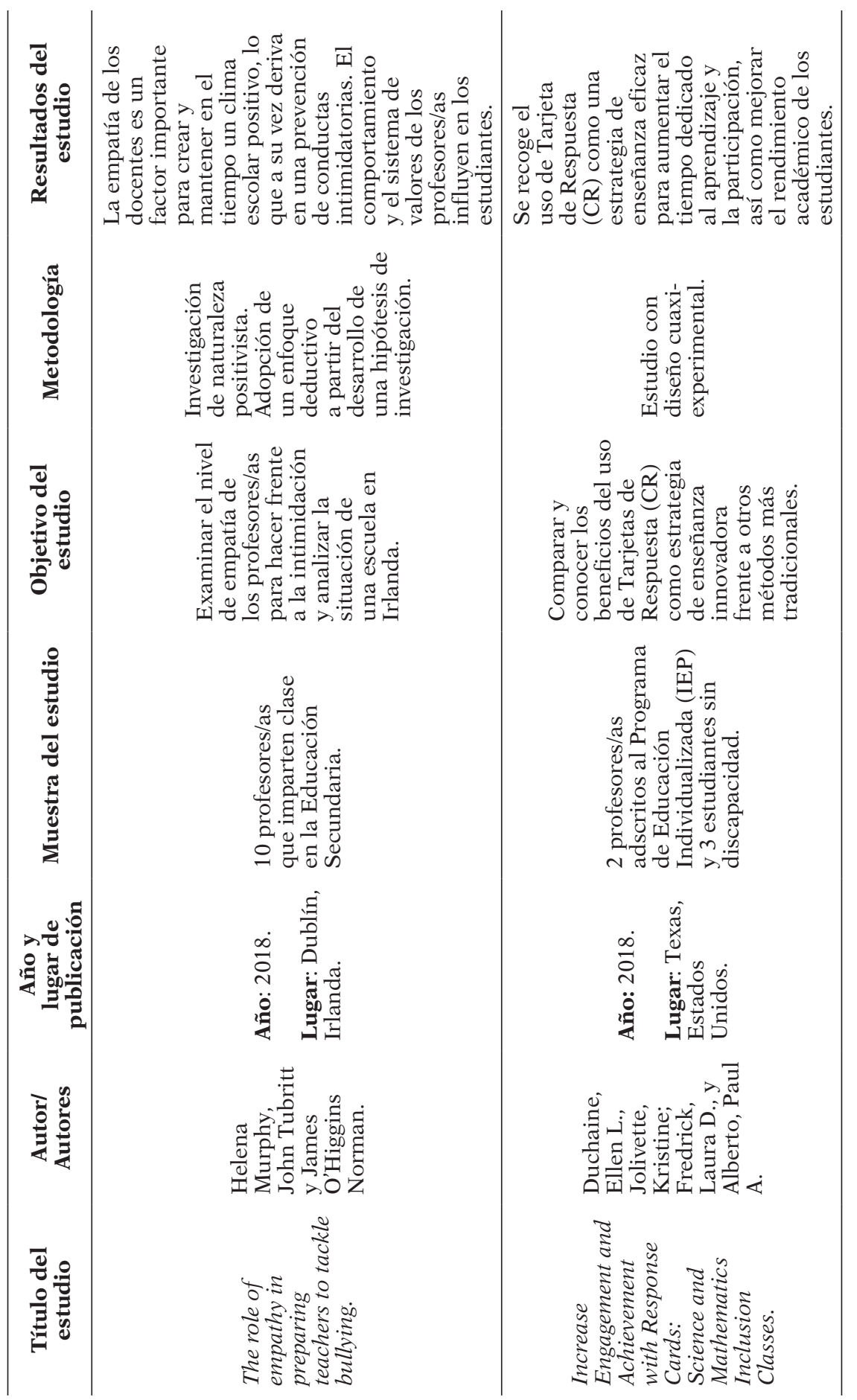




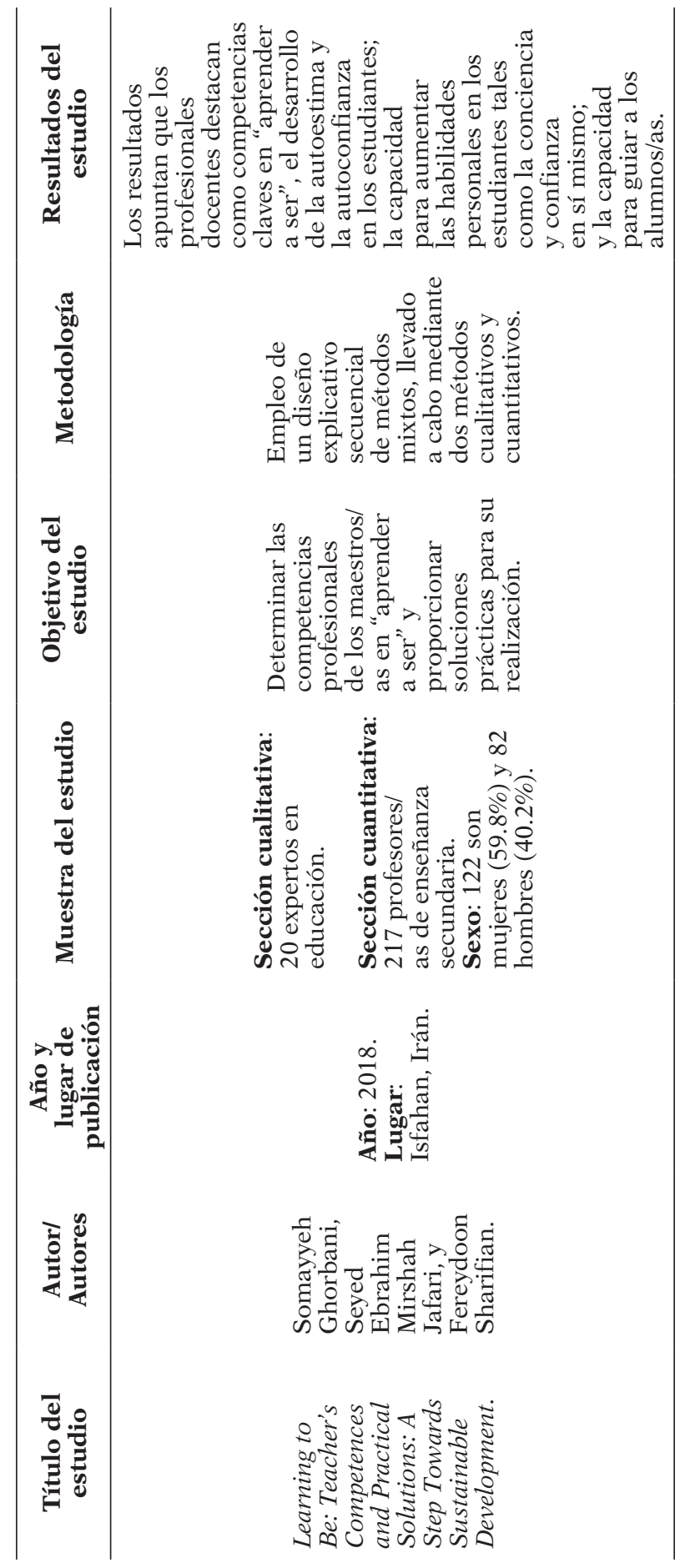




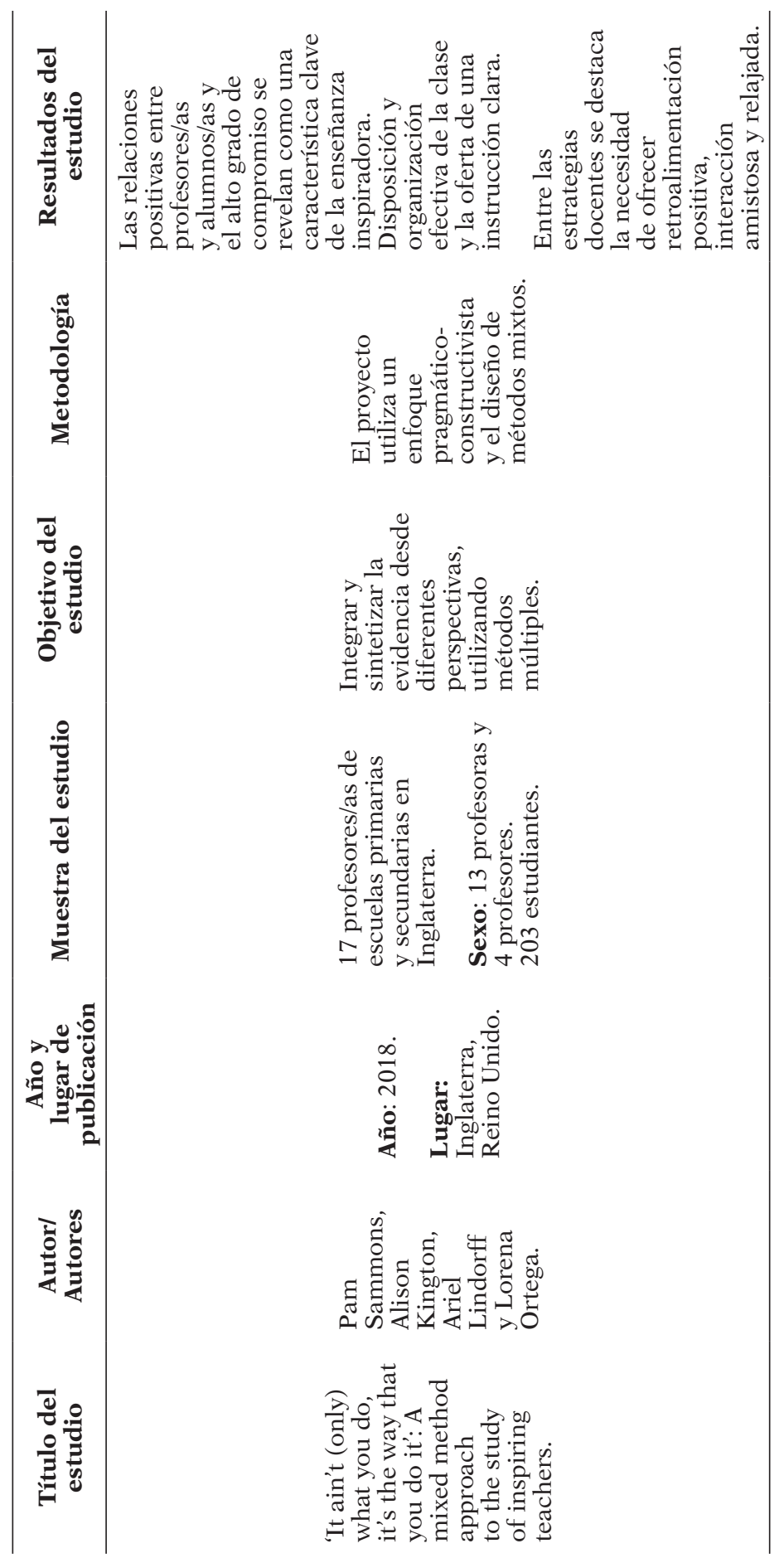




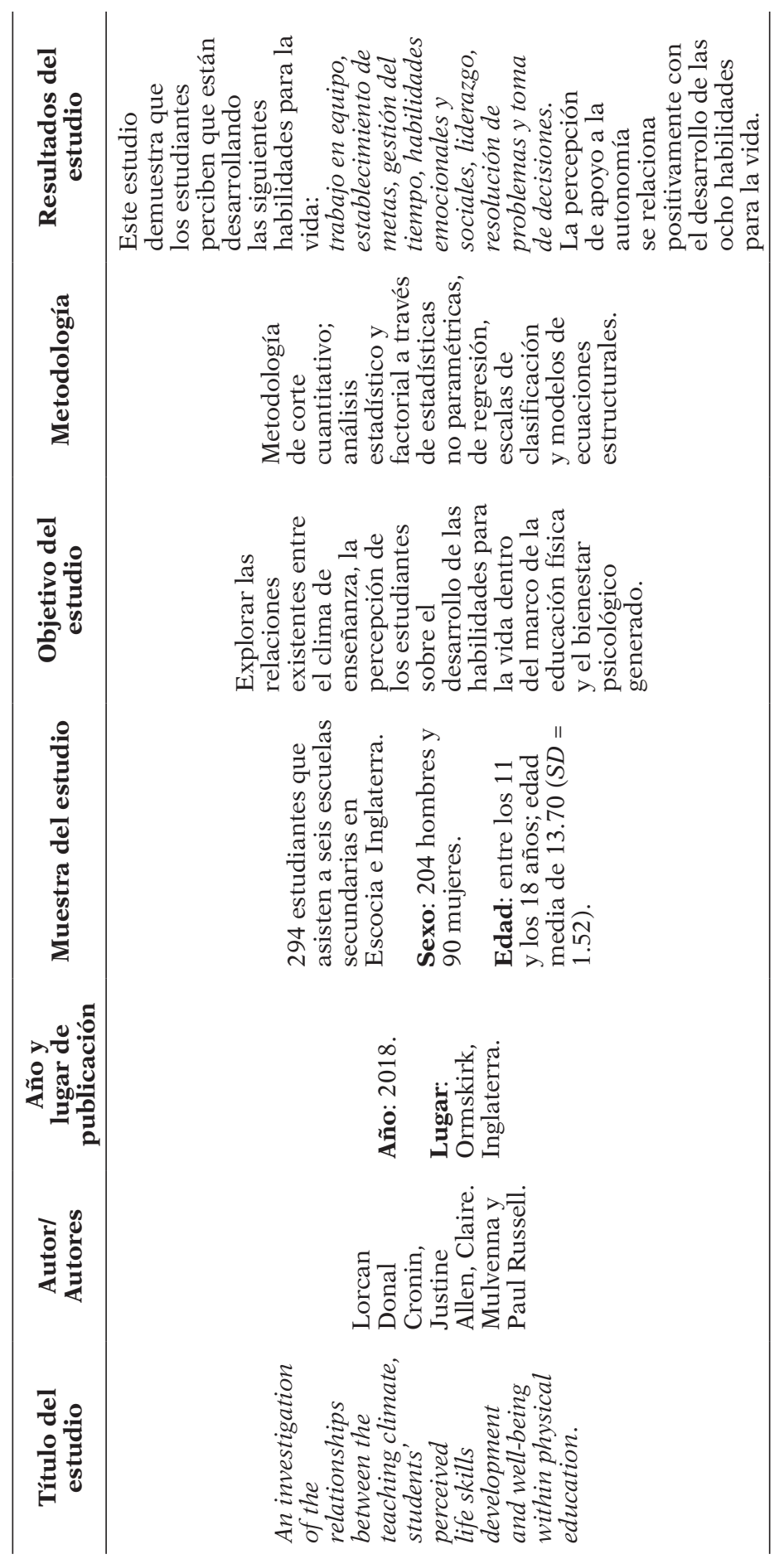




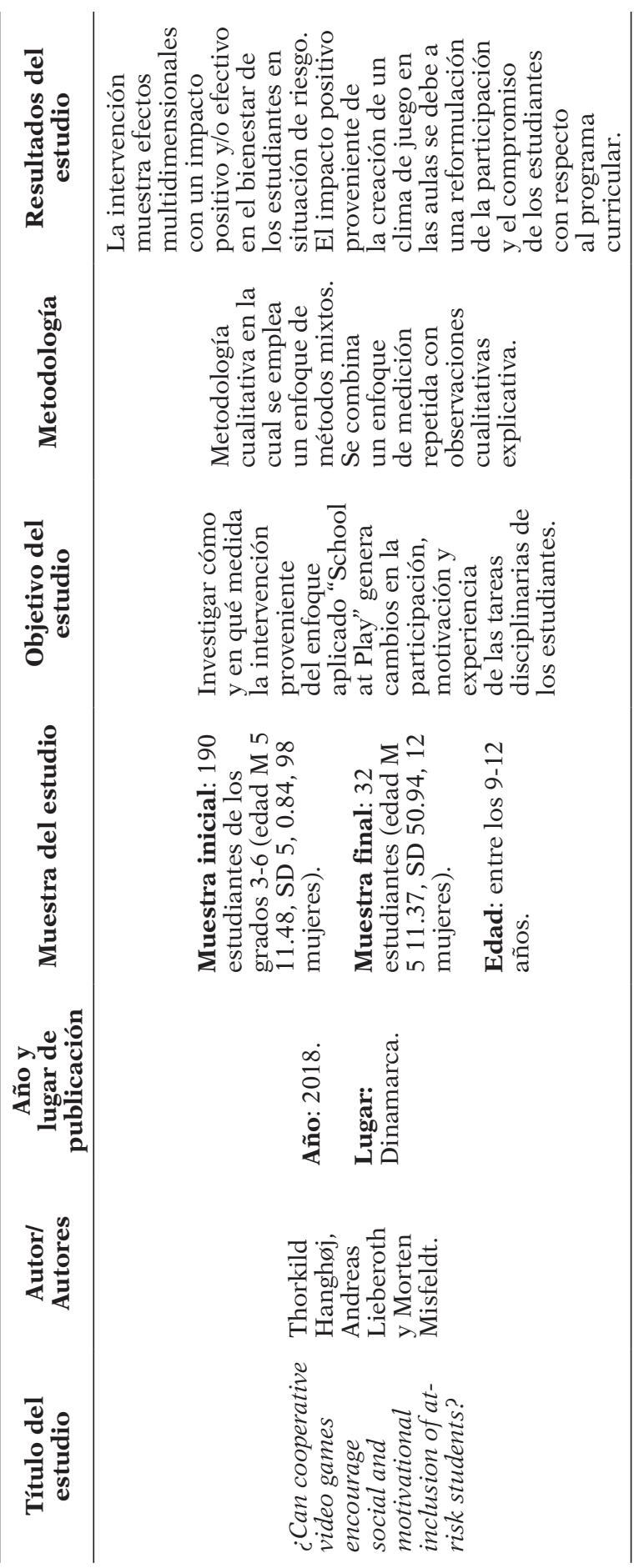

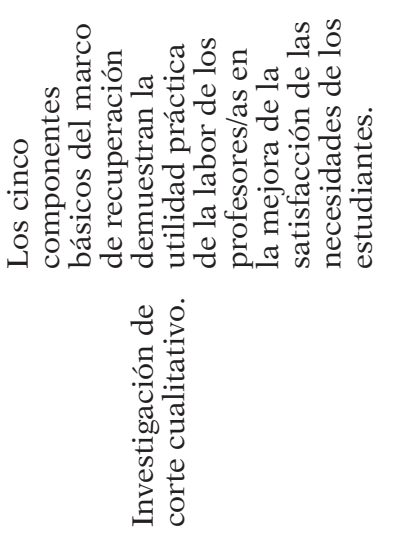
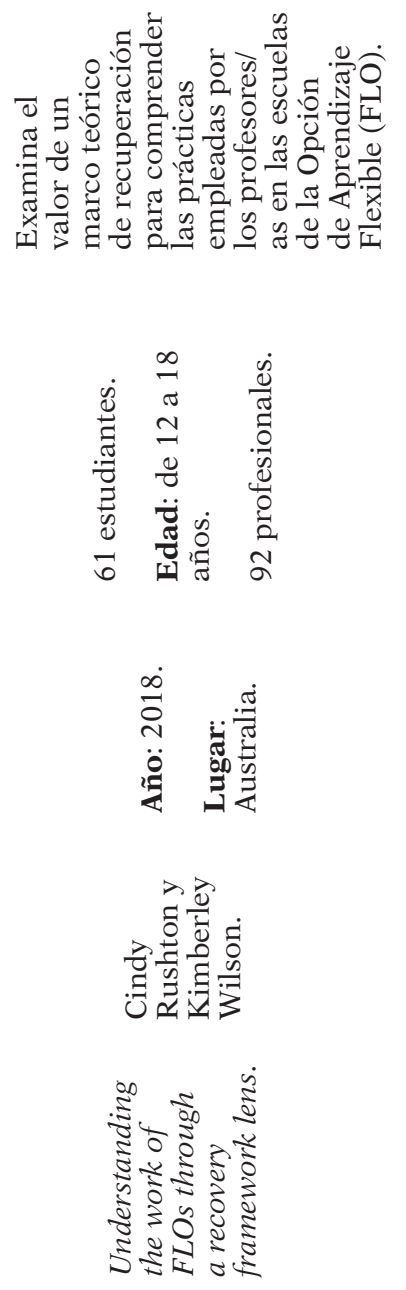


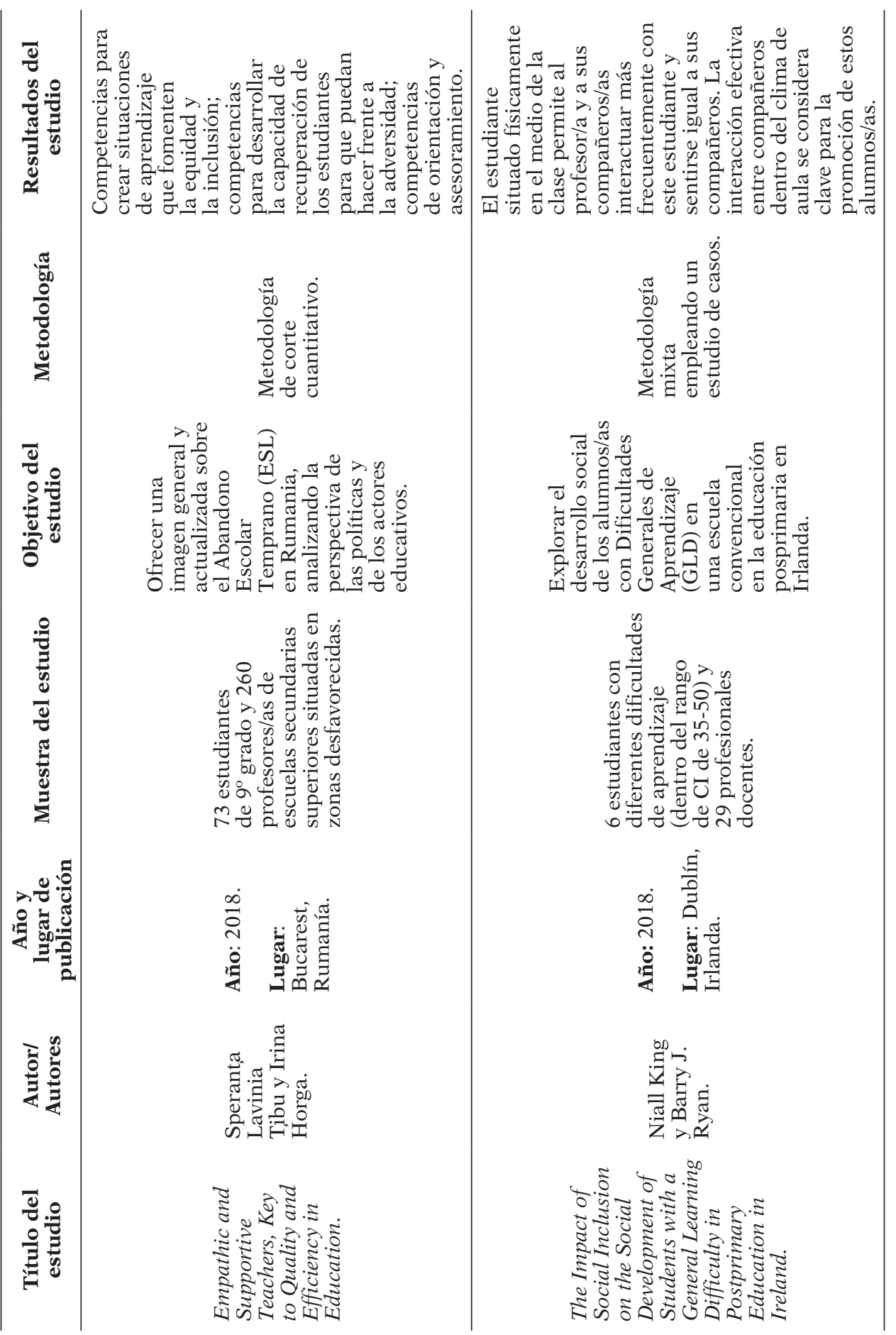




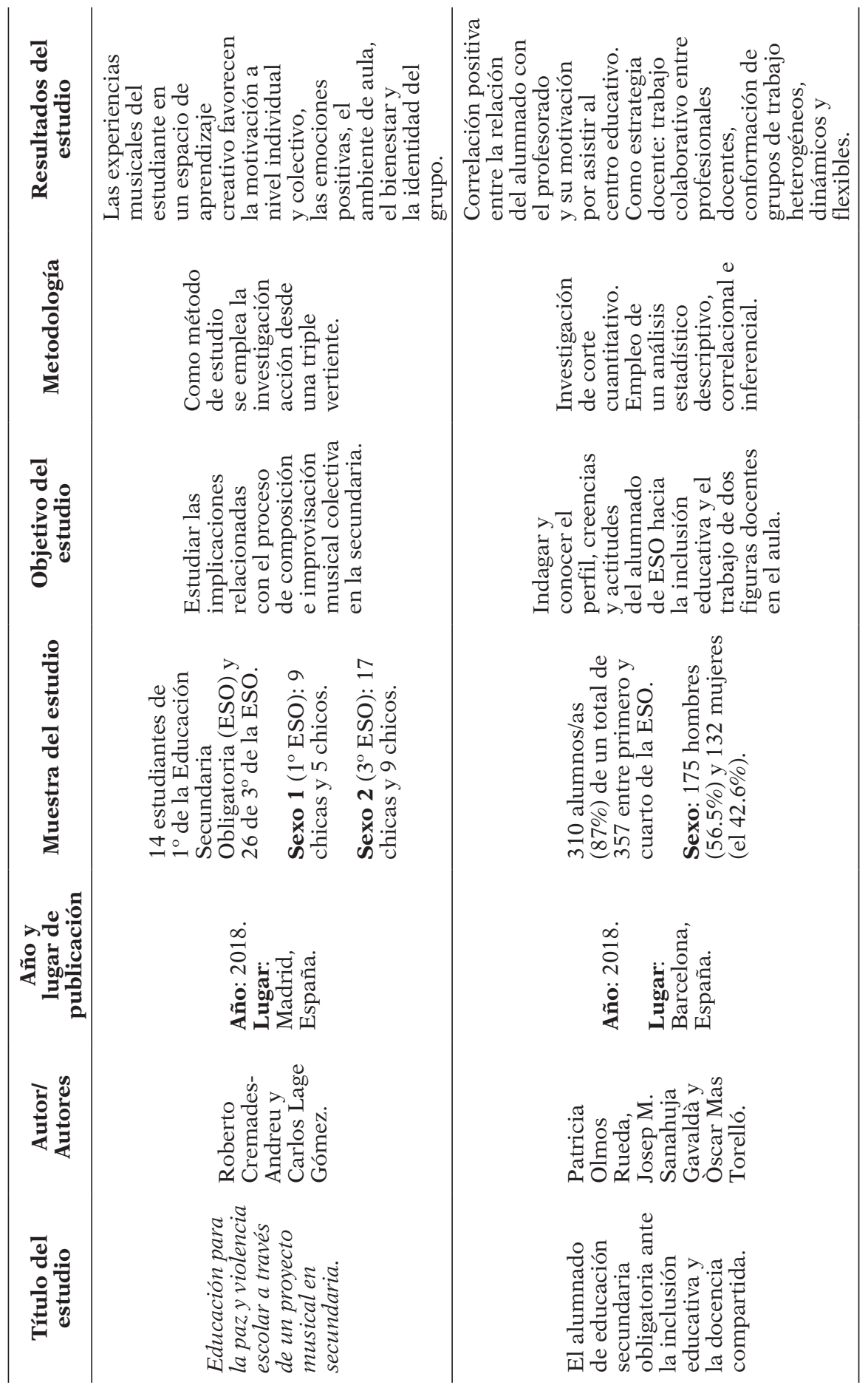




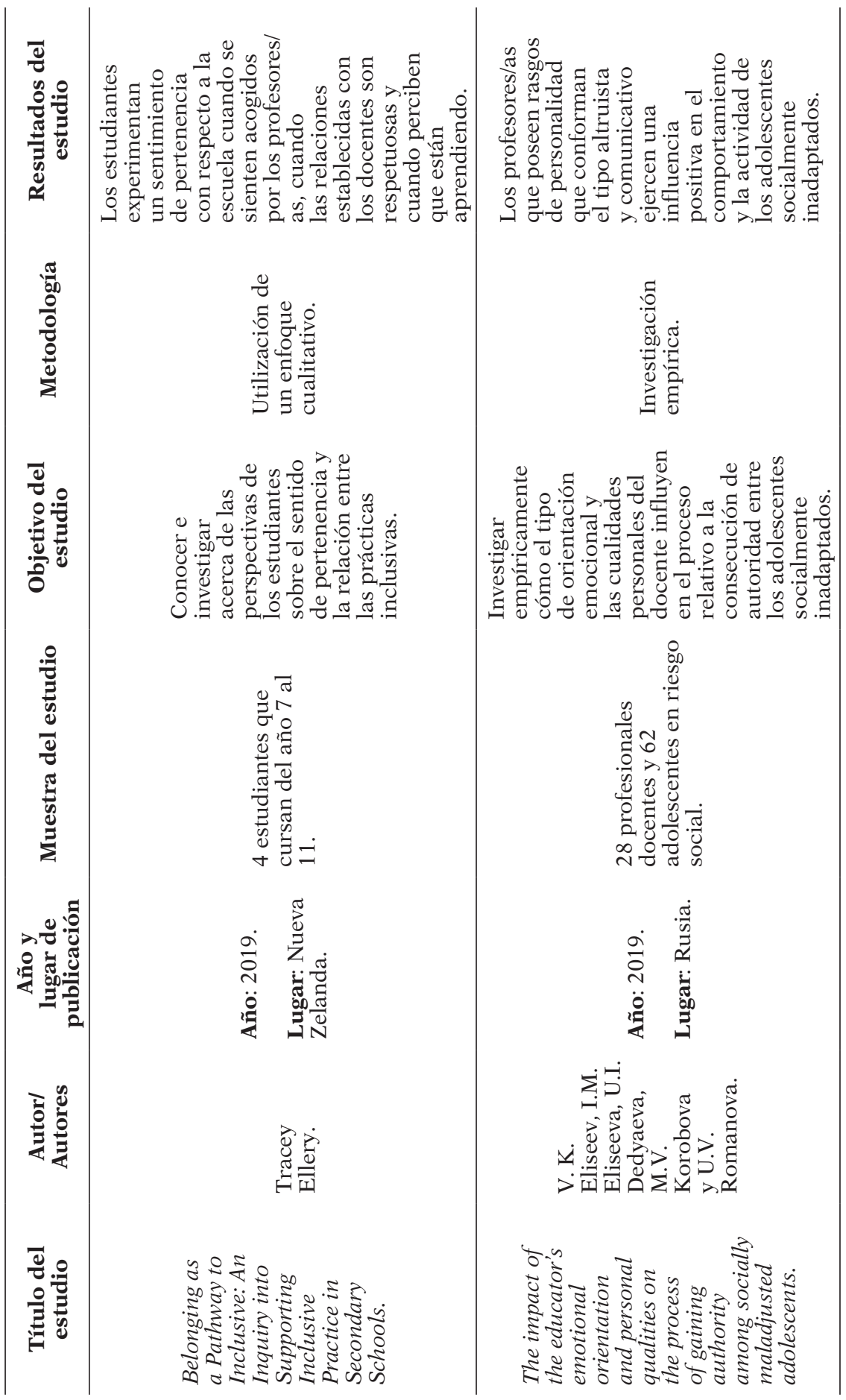




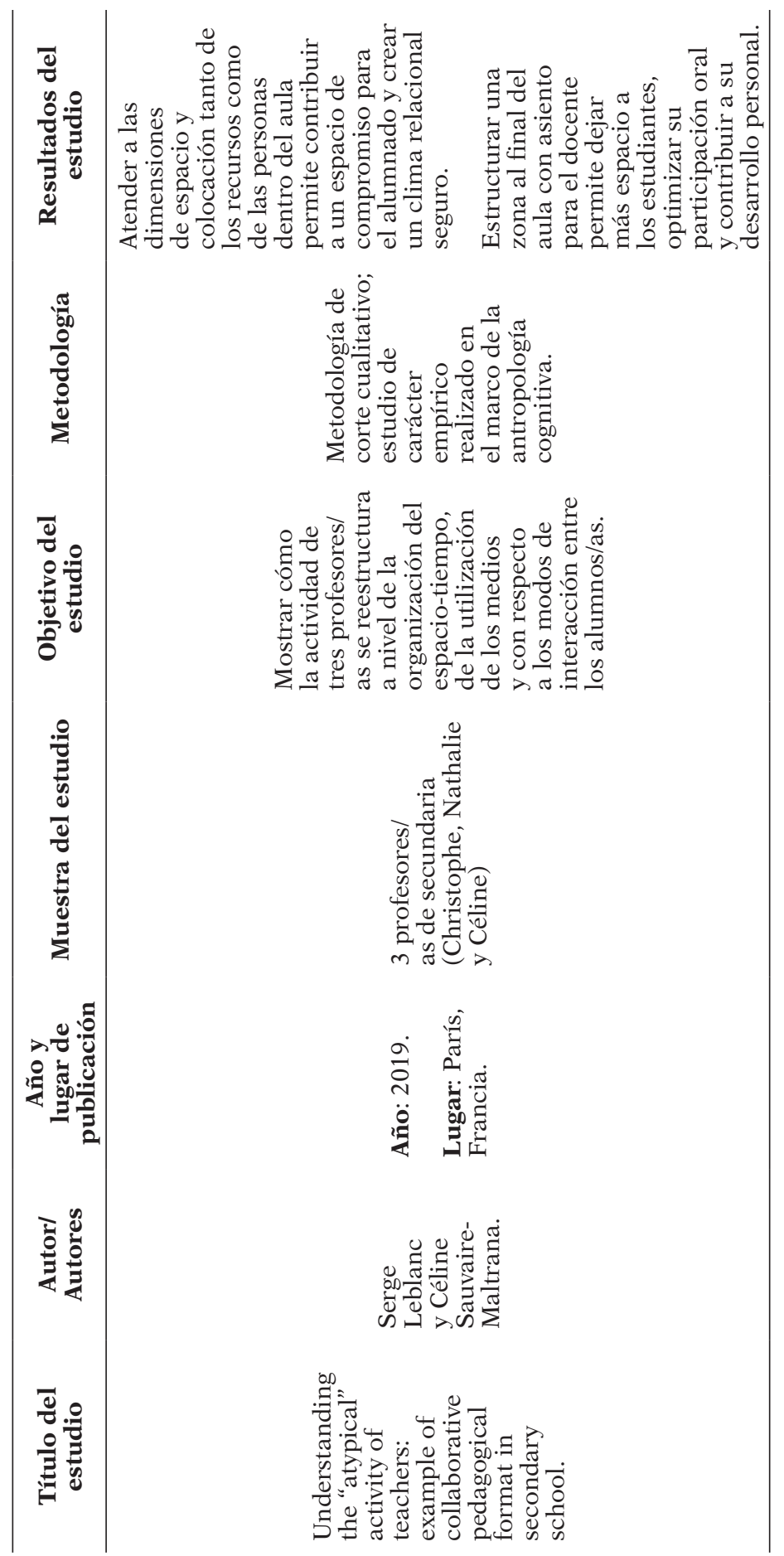




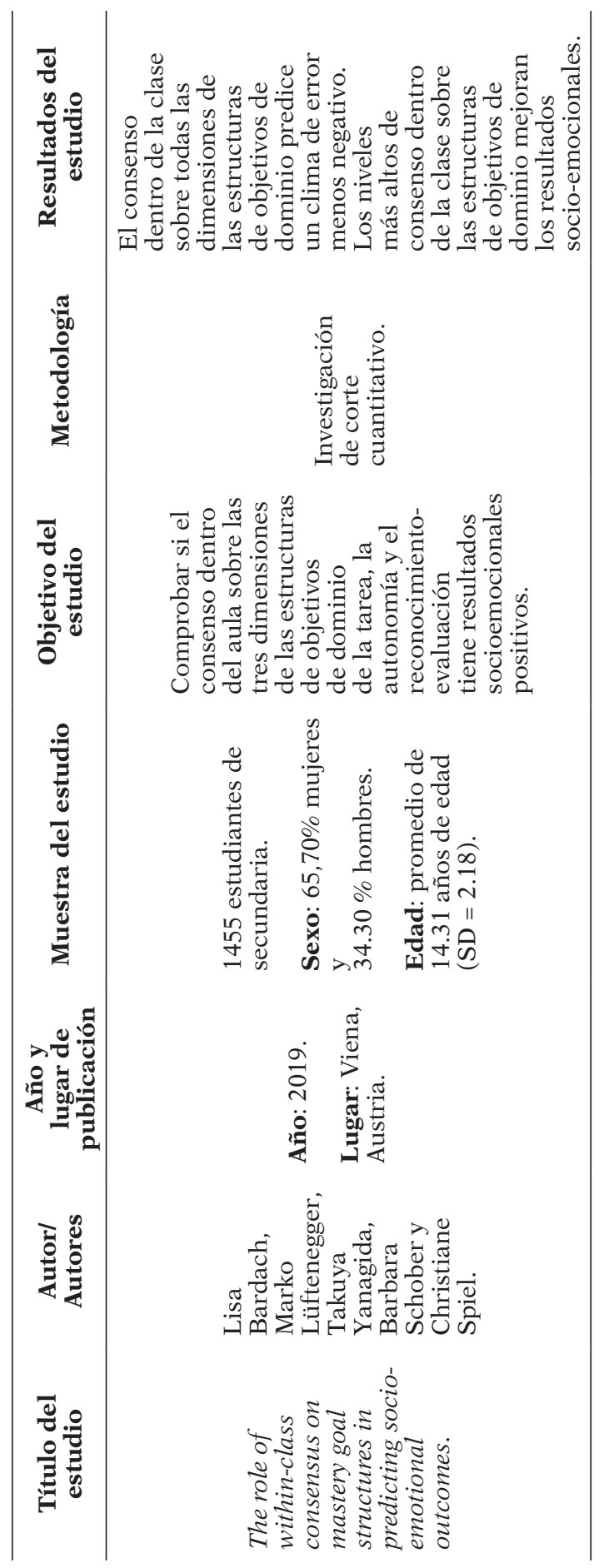




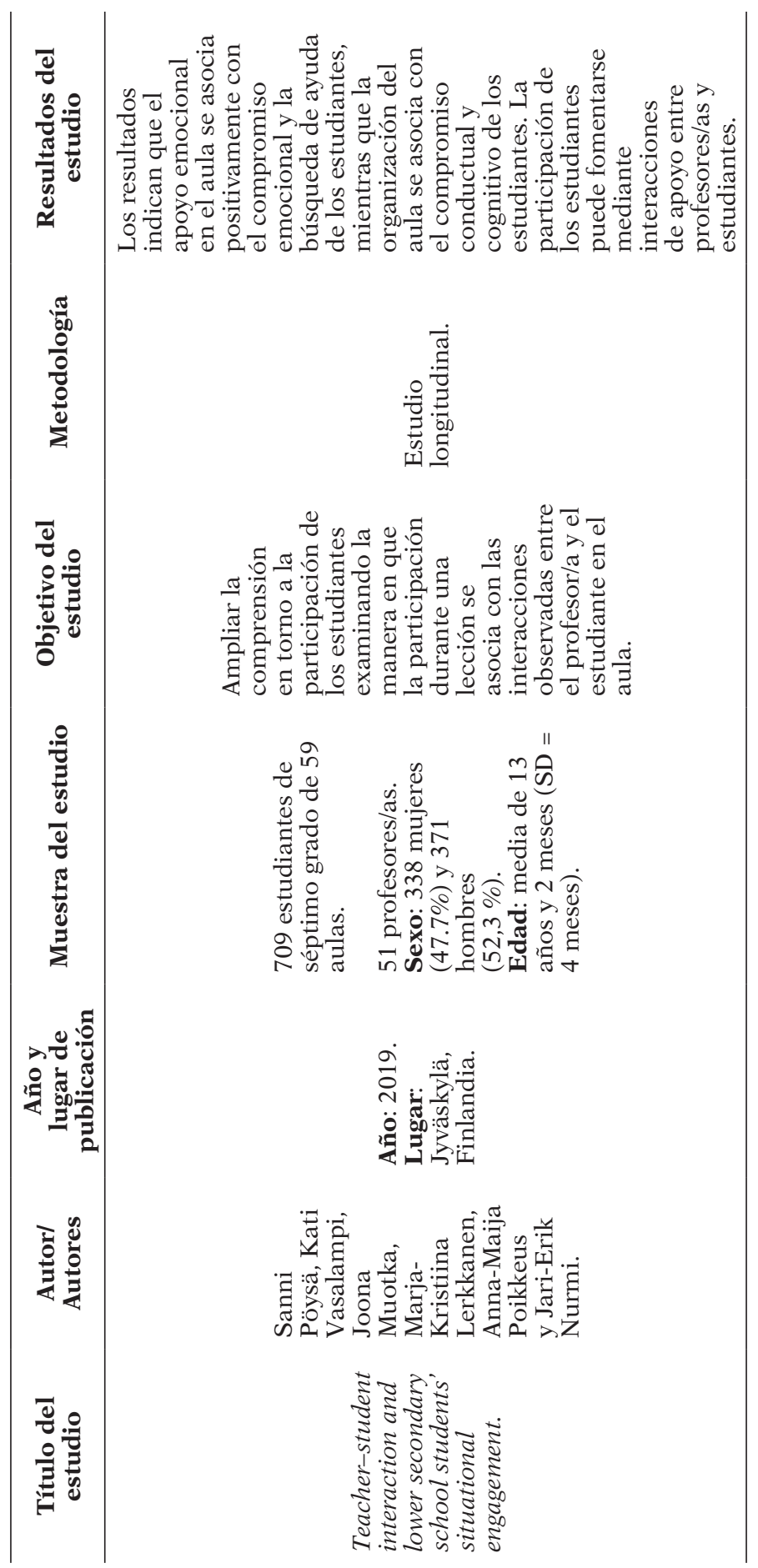




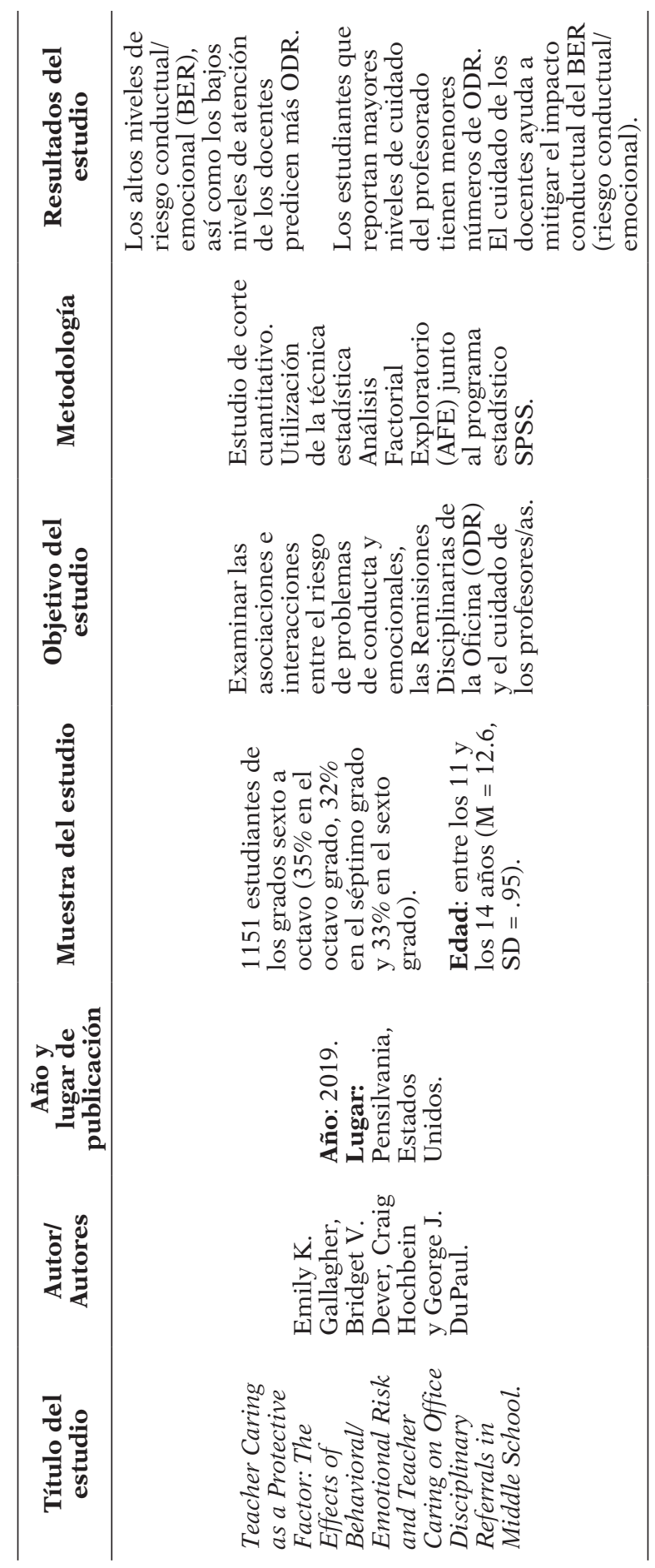




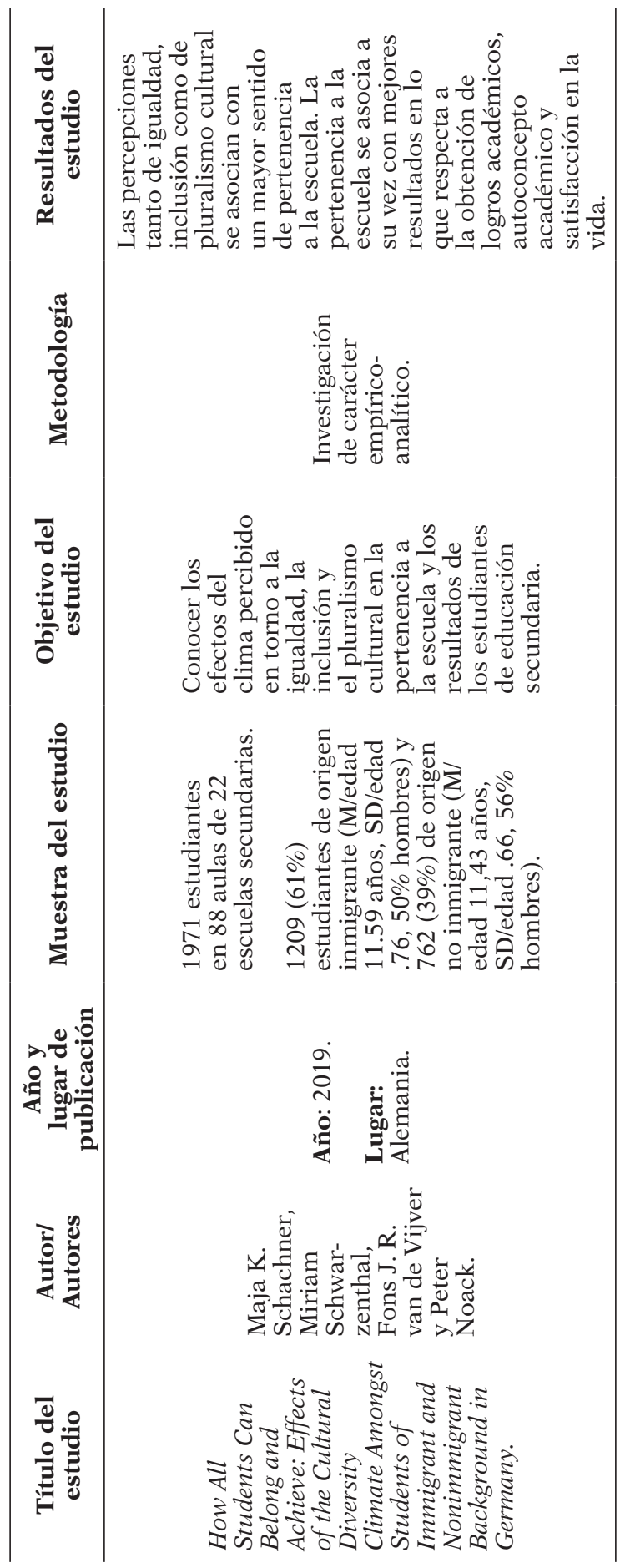




\section{PERFIL ACADÉMICO Y PROFESIONAL DE LAS AUTORAS}

Patricia Díaz Iglesias. ORCID: https://orcid.org/0000-0002-1968-2713

Graduada en Educación Infantil por la Universidad de Huelva y Máster en Psicopedagogía por la Universidad de Sevilla. Colabora con el Grupo de Investigación en Educación Emocional y Dramatización (GRieeD) de la Universidad de Sevilla. Líneas de investigación: Educación socioafectiva y ambiente educacional. Ambiente de la clase y relación profesoralumno. E-mail: patriciaiglesiasdiaz@hotmail.com

Clara Romero Pérez. ORCID: https://orcid.org/0000-0002-3159-2008

Doctora en Filosofía y Ciencias de la Educación por la Universidad de Sevilla. Máster en Pedagogía Universitaria y Enseñanza Universitaria por la Universidad de Lieja (Bélgica). Catedrática de Escuela Universitaria en la Universidad de Sevilla. Responsable del Grupo de Investigación en Educación Emocional y Dramatización (GRieeD). Líneas de investigación: Educación socio-afectiva y ambiente educacional, Teoría de la educación, Filosofía de la Educación. E-mail: clararomero@us.es

Fecha Recepción del Artículo: 19. Octubre. 2020

Fecha Modificación del Artículo: 04. Febrero. 2021

Fecha Aceptación del Artículo: 20. Febrero. 2021

Fecha Revisión para Publicación: 23. Febrero. 2021 